\title{
Applications of Fianite in Electronics
}

\author{
Alexander N. Buzynin, ${ }^{1}$ Yury N. Buzynin, ${ }^{2}$ and Vitaly A. Panov ${ }^{1}$ \\ ${ }^{1}$ A. M. Prokhorov General Physics Institute, Russian Academy of Sciences, Moscow 119991, Russia \\ ${ }^{2}$ Institute for Physics of Microstructures, Russian Academy of Sciences, Nizhny Novgorod 603950, Russia
}

Correspondence should be addressed to Alexander N. Buzynin, abuzynin@yandex.ru

Received 7 January 2012; Accepted 2 May 2012

Academic Editor: Jung Huang

Copyright (C) 2012 Alexander N. Buzynin et al. This is an open access article distributed under the Creative Commons Attribution License, which permits unrestricted use, distribution, and reproduction in any medium, provided the original work is properly cited.

Fianite or yttrium stabilized zirconia (YSZ) solid solutions single crystals were known worldwide as jewelry material. The review is devoted to novel applications of the material in the field of microelectronics. A number of modern aspects of the application of fianite in micro-, opto- and SHF-electronics were analyzed in this paper. It was demonstrated that fianite is an extremely promising multipurpose material for new electronic technologies due to unique combination of physical and chemical properties. As a substrate and buffer layer for the epitaxy of $\mathrm{Si}, \mathrm{Ge}, \mathrm{GeSi}$ and $\mathrm{A}^{\mathrm{III}} \mathrm{B}^{\mathrm{V}}$ compounds (GaAs, InGaAs, GaSb, InAs, GaN, AlN), fianite has a number of advantages over the other dielectric materials. The use of fianite (as well as $\mathrm{ZrO}_{2}$ and $\mathrm{HfO}_{2}$ oxides) instead of SiO as gate dielectrics in CMOC technology seems to be of peculiar interest. The unique properties of fianite as protecting, stabilizing and antireflecting coatings in electronics and optoelectronic devices have been outlined. A comparative study of the performance characteristics of fianite and conventional materials has been carried out.

\section{Introduction}

The further progress in electronics is connected with application of new materials. Fianite is a material of such a kind. Industrial technology of synthesis of fianite has been for the first time developed in Russia in the Lebedev Physical Institute of the Russian Academy of Sciences (FIAN in Russian), so the crystals were entitled after the Institute [1]. Serial production of the crystals has been already started in the early seventies of the twentieth century. Currently, fianite crystals are in the second position by the volume of worldwide production following silicon $[2,3]$. Fianite single crystals, -zirconia-based solid solutions (or "yttrium stabilized zirconia" YSZ), were known as jewelry stone imitation materials. Recently, in the countries with the developed microelectronics a significant growth of interest to various aspects of fianite application in semiconductor technologies has been observed. Fianite is an extremely promising multipurpose material for new optoelectronics technologies due to its unique combination of physical and chemical properties. It can be used in, virtually, all of the main technological stages of the production of electronic devices (Figure 1).
A number of application prospects of fianite in modern electronics are considered in this paper.

\section{Fianite as a Substrate and Buffer Layer for Epitaxy of Semiconductors, Multilayer Heterostructures, and Superlattices}

Appropriate conditions of growth of mirror-flat singlecrystalline films of $\mathrm{A}^{\mathrm{IV}}$ : Ge, GeSi, and $\mathrm{A}^{\mathrm{III}} \mathrm{B}^{\mathrm{V}}$ compounds: GaAs, InGaAs, GaSb, InAs, GaN, AlN, and $\mathrm{InN}$ as well as multilayer InGaAlAs heterostructures and $\mathrm{GaSb} / \mathrm{InAs}$ supperlattices on fianite substrates, as well as on $\mathrm{Si}$ and GaAs substrates coated with fianite buffer layer, using MOCVD, HW-CVD, and laser deposition techniques have been elaborated. All of these films have been for the first time synthesized in Russia.

2.1. Fianite as a Substrate and Buffer Layer for Si, Si$G e$, and $A^{I I I} B^{V}$ Compounds Epitaxy. Fianite has a number of advantages as a substrate and buffer layer at $\mathrm{Si}$ and $\mathrm{A}^{\mathrm{III}} \mathrm{B}^{\mathrm{V}}$ compounds epitaxy, as compared with other dielectric materials [4-13]. 


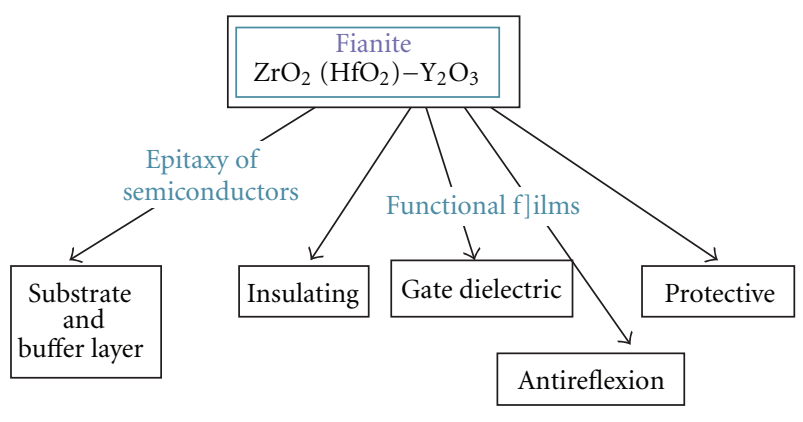

FIgUre 1: Application of fianite in electronics.

In comparison with the other dielectrics, there are the following merits of fianite in application as a substrate material and buffer layer for $\mathrm{Si}$ and $\mathrm{A}^{\mathrm{III}} \mathrm{B}^{\mathrm{V}}$ compounds epitaxy:

(i) High resistivity $\longrightarrow 10^{12} \mathrm{Ohm} \cdot \mathrm{cm}$ at $300 \mathrm{~K}$.

(ii) Similarly to $\mathrm{Si}, \mathrm{Ge}$, and $\mathrm{A}^{\mathrm{III}} \mathrm{B}^{\mathrm{V}}$ compounds, it is of cubic structure (in contrast to hexagonal of sapphire).

(iii) It is possible to alter fianite cubic lattice constant in solid solutions by varying the ratio of the main (zirconium or hafnium dioxide) and stabilizing oxides (yttria, rare earth oxides from gadolinium to lutetium, and alkaline-earth oxides) that allows an optimum matching between substrate and cubic lattice of semiconductor films thus improving its structural perfection.

(iv) Negligible value of diffusion coefficients of cations up to $1000-1200^{\circ} \mathrm{C}$ temperature range that excludes interdiffusion of impurities between the substrate and film and prevents undesirable doping (i.e., typical for sapphire), which can damage heteroepitaxial layers through penetration of impurity atoms.

(v) Due to its excellent stability at elevated temperatures, the upper limit of the corresponding structure operational temperatures depends on physical properties of a semiconductor only. Elevated temperature is not critical for the substrate.

(vi) Broad spectral range of transmission (260-7500 nm) completely covers actual absorbance and emission of Si, $\mathrm{A}^{\mathrm{II}} \mathrm{B}^{\mathrm{V}}$ compounds and its solid solutions. That makes "semiconductor-on-fianite" structures very promising for the development of various optoelectronic devices with improved operational parameters (avalanche photodiodes, light-emitting and laser diodes, etc.).

(vii) Application of thin layers of fianite on $\mathrm{Si}$ and GaAs instead of its monolithic substrates allows avoiding spatial limitations of the structures and decreasing the net cost. At the same time, the structures on "fianite/Si" and "fianite/GaAs" episubstrates have better heat conductivity in comparison with the structures on monolithic substrates.
The first epitaxial Si films on YSZ were grown in [6]. The first successful results on epitaxial MOCVD growth of various $\mathrm{A}^{\mathrm{III}} \mathrm{B}^{\mathrm{V}}$ compounds (GaAs, InAs, InGaAs, AlGaAs, GaAsN, and GaN) on YSZ are presented in a number of studies $[6,11,12]$, InN on YSZ in $[14,15]$. In $[13,16]$ a capillary epitaxy technique-the new effective way of heteroepitaxy was developed. It has been shown that the use of capillary forces in the method positively influences both on the mechanism of epitaxial growth and on quality of $\mathrm{A}_{\mathrm{III}} \mathrm{B}_{\mathrm{V}}$ epitaxial films and also reduces the minimum thickness of a continuous layer $[16,17]$.

An application of fianite as either monolithic substrate or buffer layer in "semiconductor-on-dielectric" technology is of peculiar importance for micro- and optoelectronics. The technology allows improving such characteristics of integrated circuits as operation speed, critical operational temperature, and radiation resistance.

Due to a decrease of the loss of current and stray capacitance, energy consumption of the devices is decreasing. Moreover, the devices based on "semiconductor-ondielectric" structures are more reliable, especially under extreme operational conditions. Currently, "silicon-oninsulator" structures are one of the most dynamically developing directions in the field of semiconductor materials science. However, electrophysical and operational parameters of the devices as well as its radiation resistance and reliability significantly suffer because of structure imperfection of silicon layers. In case of "silicon-on-sapphire" structures, the imperfection is determined, in particular, by a difference in crystallographic structure of silicon and sapphire, as well as by autodoping of a silicon film by aluminum penetrating from the sapphire substrate in concentrations up to $10^{18}-$ $10^{20} \mathrm{~cm}^{-3}$. Considering crystal-chemical and physical characteristics of fianite, the material is more preferential for the epitaxy of $\mathrm{Si}$ as an alternative substrate in comparison with sapphire.

2.2. Silicon-on-Fianite Epitaxial Structures. The first studies on silicon epitaxy on fianite single-crystal substrates have been carried out in France and USA [6, 7]. Silicon films on fianite substrate were deposited by chloride and hydride epitaxy at $900-1100^{\circ} \mathrm{C}$. The films obtained were of polycrystalline structure and, consequently, of rather poor electrophysical parameters. However, at the same time, it was shown that silicon-on-fianite structures sustaining actually all advantages of silicon-on-sapphire are free from its principal drawbacks.

At the epitaxy of $\mathrm{Si}$ on fianite, a formation of $\mathrm{SiO}_{2}$ intermediate layer between the film and the substrate was observed $[7,8]$. Subsequent annealing of the structure led to the increase of $\mathrm{SiO}_{2}$ layer thickness. It was demonstrated [8] that the layer can improve properties of silicon-on-fianite epitaxial structure because its formation:

(i) removes mechanical stress in the layer-substrate interface; 
(ii) smoothens over negative effect occurring due to a difference of linear expansion coefficients between fianite and silicon;

(iii) improves insulation of the integrated circuit elements (ICE) based on Si;

(iv) acts as a barrier for metal impurities diffusing from the substrate and forming deep levels in silicon.

The formation of $\mathrm{SiO}_{2}$ intermediate layer at hightemperature epitaxy is associated with peculiar properties of fianite. In contrast to the other dielectrics, fianite features with a unique peculiarity as a solid electrolyte: starting from $650^{\circ} \mathrm{C}$, it becomes actually oxygen-transparent due to high mobility of oxygen. The reason for significant mobility of oxygen in fianite crystals is an occurrence of oxygen vacancies due to $\mathrm{Zr}^{+4}$ to $\mathrm{Y}^{+3}$ cation substitution at formation of the solid solution. High mobility of oxygen in fianite crystals is determined by an occurrence of oxygen vacancies at $\mathrm{ZrO}_{2}\left(\mathrm{HfO}_{2}\right)-\mathrm{R}_{2} \mathrm{O}_{3}$ (here: $\mathrm{R}-\mathrm{Y}, \mathrm{Gd}-\mathrm{Yb}$ ) solid solutions formation due to $\mathrm{Zr}^{+4}\left(\mathrm{Hf}^{+4}\right)$ to $\mathrm{R}^{+3}$ cation substitution. The process results in oxygen nonstoichiometric $\mathrm{ZrO}_{2}\left(\mathrm{HfO}_{2}\right)$ based phase [4]. Because of the high mobility of oxygen at high temperature of the epitaxy $\left(900-1000^{\circ} \mathrm{C}\right)$ used in [6-8], the formation of ether $\mathrm{SiO}_{2}$ continuous layer or its islets between the substrate and the film was shown to be inevitable.

The phenomenon occurs even at the epitaxy initial stages when a continuous epitaxial film is forming. It was shown [9] that the formation of $\mathrm{SiO}_{2}$ layer or isles at the initial stage of molecular-beam epitaxy on fianite results in 3-dimensional mechanism of growth, formation of structural defects and hindered the synthesis of Si films of single-crystal structure. The occurrence of the isles at the initial epitaxy stages and the polycentric growth of Si layers were shown possible to avoid only by using a set of techniques, those which prevent diffusion of oxygen from the substrate to the film at the initial stage of the process. In particular, high structural perfection of the Si-on-fianite films was achieved by using a low-temperature $\left(T<650^{\circ} \mathrm{C}\right)$ molecular-beam epitaxy [7].

2.3. Ge and GeSi Films on Fianite Substrates. Growth of $\mathrm{Ge}$ and $\mathrm{Ge}-\mathrm{Si}$ heterostructures on fianite substrates was carried out using HWCVD installation. Base pressure in the chamber $\sim 1 \cdot 10^{-8}$ torr was maintained by pumping-down using two heteroionic pumps. A high-vacuum gate was used for isolation of the growth cell and the pumps from other parts of the vacuum system. Forepumping of the chamber was performed using a diffusion pump. The diffusion pump allowed to exhaust any gas (including $\mathrm{GeH}_{4}$ ) both in atomic and molecular state. FM-1 oil with low vapor pressure was used as a pressure fluid. There was a nitrogen trap above the diffusion pump preventing reverse diffusion of the oil from preevacuation and diffusion pumps into the growth cell. The (100) and (111) oriented fianite single-crystal plates were used as substrates. Silicon atomic beam was maintained by sublimation of the element single-crystal (high resistance) in form of $4 \times 4 \times 90 \mathrm{~mm}$ ingot sections. The sources were mounted on the cooled current leads. There was a Ta plate of $80 \times 5 \times 0.5 \mathrm{~mm}$ size installed in one of the sources position.

Before the epitaxial growth, the sources and substrates were subjected to $10 \mathrm{~min}$ annealing at 1350 and $1250^{\circ} \mathrm{C}$, respectively, then temperature of the source was increased to $1380^{\circ} \mathrm{C}$, as the substrate temperature was decreased to assigned values $\left(600-700^{\circ} \mathrm{C}\right)$ and the buffer layer was grown. The pressure in the cell corresponded to basic one.

In order to grow Ge layers, the cell was filled with $\mathrm{GeH}_{4}$ up to $1 \cdot 10^{-3}-5 \cdot 10^{-6}$ torr and the pressure was maintained constant by a system of the gas feeding. Simultaneously, the Ta plate situated in vicinity of the substrate was heated to $T=1200^{\circ} \mathrm{C}$. With the purpose to avoid destruction of germane on evaporators ( $\mathrm{Ti}$ ) following preepitaxial annealing of the sources and substrates, the sublimating pumps were switched off and the growth was carried out at pumping down using only diffusion- and booster-pumps. It is worth to note that the gas filling up to such high pressure $\left(\sim 10^{-3}\right.$ torr $)$ is impossible in MBE installations with electron-beam heating. Germane pressure in the cell was tentatively assigned by ionization vacuum gage indications. Nevertheless, this peculiarity in $\mathrm{GeH}_{4}$ pressure measurement did not impede the controlled growth of Ge films at $700-750^{\circ} \mathrm{C}$ temperature of the substrate. The films were continuous and homogeneous. GeSi solid solutions with up to $80 \% \mathrm{Si}$ content were also obtained on (111) and (100) fianite substrates. Vacuum annealing at $1250^{\circ} \mathrm{C}$ during $10 \mathrm{~min}$ was used as a preepitaxy treatment. The growth was carried out under $5 \cdot 10^{-4}$ torr germane pressure and at $600^{\circ} \mathrm{C}$ substrate temperature. Simultaneously, the Ta plate positioned in vicinity of the substrate was heated to $1200^{\circ} \mathrm{C}$. The heteroepitaxial Ge films obtained show high structural perfection. X-ray rocking curve (XRC) FWHM values were $0.31^{\circ}$ for Ge film. The surface morphology of the Ge epitaxial layers grown on (100) and (111) fianite substrates as well as the peaks of Raman scattering near $300 \mathrm{~cm}^{-1}$ are identical to those of bulk Ge. Therefore, it is possible to conclude that there are no stains in the Ge/fianite layer.

2.4. Epitaxial Films of $A^{I I I} B^{V}$ on Fianite. Crystallochemical and physical properties of fianite are favorable not only for silicon but also for $\mathrm{A}^{\mathrm{III}} \mathrm{B}^{\mathrm{V}}$ compounds epitaxy (Table 1).

First successful results on growth of $\mathrm{A}^{\mathrm{III}} \mathrm{B}^{\mathrm{V}}$ compound epitaxial films on fianite substrates were presented in [10, 18]. GaAs, InAs, GaN, and other $\mathrm{A}^{\mathrm{III}} \mathrm{B}^{\mathrm{V}}$ semiconductor compound films have been grown on fianite, as well as on silicon and gallium arsenide with fianite buffer layer substrates by means of metal-organic chemical vapor deposition (MOCVD). A new efficient epitaxy technique-"capillary epitaxy" has been suggested. The technique allowed synthesizing of $\mathrm{A}^{\mathrm{III}} \mathrm{B}^{\mathrm{V}}$ compound films by a MOCVD on fianite substrates. Samples of structurally perfect submicron (up to $0.1 \mu$ ) epitaxial films of $\mathrm{A}^{\mathrm{III}} \mathrm{B} \mathrm{V}$ compounds have been obtained using this technique. The samples demonstrated high electrophysical parameters [13, 17-20]. In [21], GaN epitaxial films have been grown on fianite substrates by MOVP technique. It was observed that the epitaxial growth 
TABLE 1: Some properties of fianite crystals and $\mathrm{A}^{\mathrm{III}} \mathrm{B}^{\mathrm{V}}$ compounds.

\begin{tabular}{|c|c|c|c|c|c|}
\hline \multirow[t]{2}{*}{ Crystal } & \multicolumn{2}{|c|}{ Lattice } & \multirow{2}{*}{$T_{m},{ }^{\circ} \mathrm{C}$ (melting point) } & \multirow{2}{*}{ Thermal expansion coefficients $10^{-6} \mathrm{deg}^{-1}$} & \multirow{2}{*}{$E_{g}, \mathrm{eV}$} \\
\hline & Type & $a, \AA$ & & & \\
\hline$\left(\mathrm{ZrO}_{2}\right)_{100-x}\left(\mathrm{Y}_{2} \mathrm{O}_{3}\right)_{x}$ & Cubic (fluorite) & $\begin{array}{l}5.141(x=10) \\
5.157(x=15) \\
5.198(x=21)\end{array}$ & 2800 & $11.4\left(15-1000^{\circ} \mathrm{C}\right)$ & \\
\hline GaAs & Cubic (sphalerite) & 5.65 & 1283 & 5.4 & 1.43 \\
\hline $\mathrm{GaP}$ & Cubic (sphalerite) & 5.445 & 1467 & 4.7 & 2.26 \\
\hline $\mathrm{GaN}$ & Hexagonal (wurtzite) & $\begin{aligned} a & =3.186 \\
c & =5.178\end{aligned}$ & 1700 & $5.6 ; 7.8$ & 3.4 \\
\hline $\mathrm{GaN}$ & Cubic (sphalerite) & 4.52 & 1700 & 3.9 & 3.2 \\
\hline InN & Hexagonal (wurtzite) & $\begin{aligned} a & =3.54 \\
c & =5.70\end{aligned}$ & 1200 & 12.7 & 0.7 \\
\hline $\operatorname{InN}$ & Cubic (sphalerite) & 4.98 & 1200 & 4.4 & 0.67 \\
\hline
\end{tabular}

of $\mathrm{GaN}$ on fianite significantly depends on conditions of the initial stage of the process.

In $[11,22,23]$, fianite substrates were successfully tested for growth of InN heteroepitaxial films. InN films of cubic structure have been grown on (001) fianite substrates by plasma-stimulated molecular-beam epitaxy (RF-MBE) at $400-490^{\circ} \mathrm{C}$ temperature. The lattice mismatch of $\mathrm{InN}$ and fianite at (001) plane is very low (less than 2.3\%), in contrast to $17 \%$ for InN sapphire and more than $10 \%$ for InN-GaAs. Due to this fact, InN films grown on (001) fianite substrate were superior InN films grown on sapphire [10] and (001) GaAs substrates by its crystallographic perfection [15].

Therefore, fianite is apparently in advance as a substrate for InN epitaxy as compared to sapphire. A new effective method of heteroepitaxy, capillary epitaxy, was proposed in [17]. In particular, this technique allows us to obtain the films of $\mathrm{A}^{\mathrm{III}} \mathrm{B}^{\mathrm{V}}$ compounds on fianite using a MOCVD approach.

2.4.1. Deposition of GaAs, GaSb, GaAs:Sb Films, and GaSb/ InA Superlattice on Fianite Substrates by Means of Laser Sputtering. Our experiments have shown that the conventional "direct" growth of heteroepitaxial InGaAs films on fianite substrates resulted in the films with rough surface. So the buffer layers were elaborated to improve the results. The buffer layer must have very high structural perfection and mirror-homogeneous surface. A number of experiments were conducted for growth of GaAs, GaSb, and GaAs buffer layers on fianite (100) and (111) substrates as well as $\mathrm{GaSb} / \mathrm{InAs}$ superlattice by using laser sputtering. This superlattice is working as a filter, which prevents penetration of the defects into InGaAs film and, first of all, formation of growing dislocation. Furthermore, $\mathrm{Sb}$ is an effective surfactant which significantly improves the films morphology.

The studies have shown that it was complicated to obtain thin and homogeneous layers of $\mathrm{A}^{\mathrm{III}} \mathrm{B}^{\mathrm{V}}$ compounds on fianite substrates. It may be related to rather high mismatching of the lattice parameters of fianite and $\mathrm{A}^{\mathrm{III}} \mathrm{B}^{\mathrm{V}}$ compounds leading to growth according to the Volmer-Weber mechanism. Formation of the continuous layer occurred through 3-dimensional nuclei, their subsequent growth and joining. Low nuclei density results in the formation of highly inhomogeneous rough surface that hinders subsequent formation of a flat film. A laser sputtering technique is considered to maintain high nuclei density; so, before joining, the nuclei are of sufficiently small size that promotes the formation of a flat continuous film.

Therefore, in order to obtain flat layers, a laser sputtering technique was used in the study.

The Q-switched Nd laser and single-crystal GaAs and InAs targets were used. The superlattices were grown by optical switching of the laser beam between the targets. Mirror-flat GaSb and GaAs:Sb layers as well as pentaperiodic InAs/GaSb supperlattices of $0.15 \mu \mathrm{m}$ total thickness were deposited using this technique.

The X-ray diffraction investigations of GaAs:Sb (111) films on fianite (111) showed their single-crystal structure (Figure 2(a)). It was shown that the spectral dependence of photoconductivity of GaSb films on fianite substrates (Figure 2(b)) has a maximum of photoconductivity at the edge of fundamental absorption. This effect may be due to high velocity of the surface recombination.

The X-ray rocking curve (XRC) FWHM value was $0.23^{\circ}$ for GaSb (111) film. The image of the surface of GaAs:Sb $(0.2$ $\mu \mathrm{m}$ thickness) on fianite is shown in Figure 3(a). It is apparent that the surface of the layer is mirror flat and sufficiently homogeneous. The microrelief of the layer surface is shown in Figure 3(b). According to our estimations, roughness of the layer is less than $4 \mathrm{~nm}(\mathrm{Sq}=0.003778 \mu \mathrm{m})$.

In the penta-periodic InAs/GaSb supperlattices of $0.15 \mu \mathrm{m}$ total thickness grown on (111) fianite substrates electron mobility approaches to $580 \mathrm{~cm}^{2} / \mathrm{V} \times \mathrm{s}$. The $\mathrm{GaSb}$ layers, as well as InAs/GaSb short-period supperlattices, are suitable for the development of IR detectors operating in a 2-3 $\mu \mathrm{m}$ range. In our studies, the structures were used as buffers for $\mathrm{A}^{\mathrm{III}} \mathrm{N}$ growth on fianite substrates. 


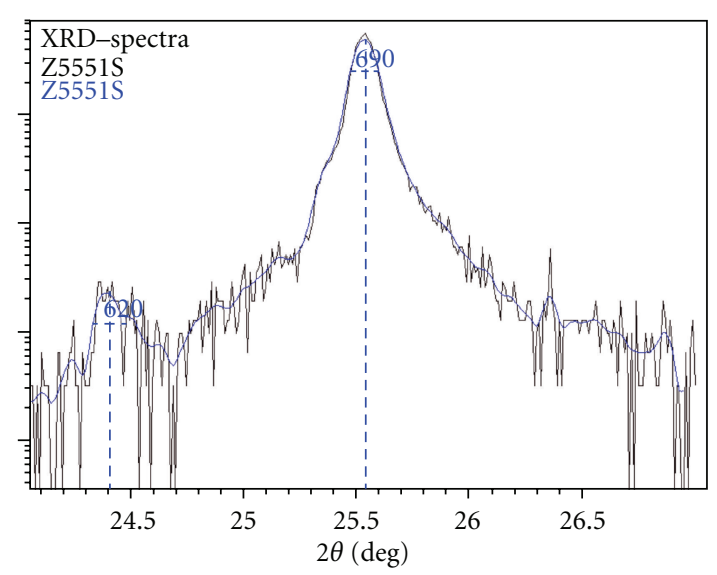

(a)

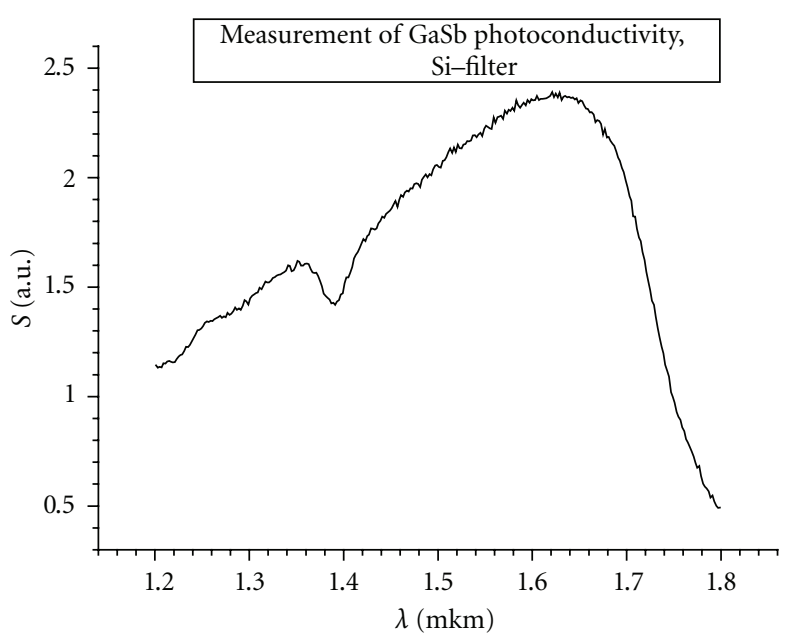

(b)

FIgURE 2: XRD $\theta / 2 \theta$ scan of GaSb (111) film on fianite (111) (a) and photoconductivity of GaSb film on the fianite substrate (b).

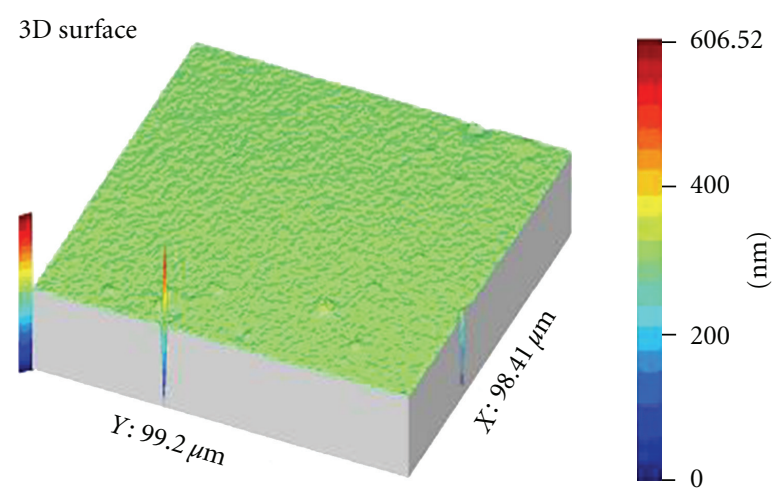

Azimuth: $66.3(\mathrm{deg})$; elevation: $40.7(\mathrm{deg})$ $X$ scale: 1 ; $Y$ scale: $1 ; Z$ scale: 1

(a)

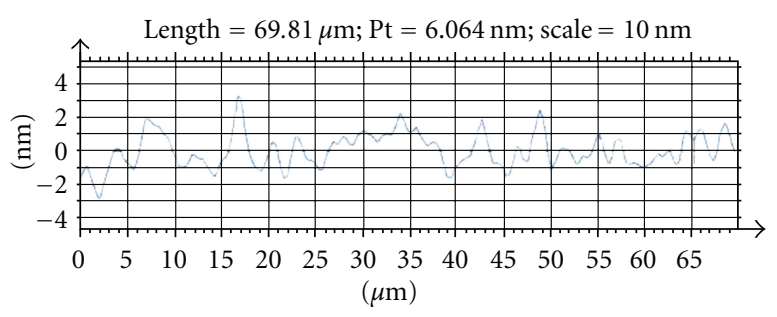

(b)

FIGURE 3: Image of surface (a) and the surface relief (b) of GaAs:Sb buffer on fianite.

2.4.2. MOCVD Capillary Epitaxy of III-V Compounds on Fianite. The investigations showed that continuous GaAs films on fianite can be obtained only in a very narrow range of the epitaxial conditions. In particular, a temperature range of $550-600^{\circ} \mathrm{C}$ is necessary. The minimum thickness of a continuous layer was $1.5-2.0 \mu \mathrm{m}$. The epitaxial films had polycrystalline structure and rough surface. Structural and electrical properties of GaAs films could be improved using capillary epitaxy. The essence of this method is that a thin (less than $50 \mathrm{~nm}$ ) film of an III-group element is initially deposited on fianite surface and then saturated with a V-group component with the formation of a thin continuous epitaxial III-V layer. Following this procedure, the film growth continues to obtain the necessary thickness under conventional epitaxial conditions.

The use of capillary forces in the first (heteroepitaxial) stage of GaAs film formation led to improvement of epitaxial quality. Electron microscopy of the GaAs films at the initial growth stages showed that the transition from conventional MOCVD growth to capillary epitaxy leads to a change in the growth mechanism. Three-dimensional island mechanism changes to the two-dimensional one with propagation of the growth steps (Figure 4(a)). This process is similar to graphoepitaxy $[24,25]$ from aqueous solutions with addition of surfactants, where an increase in the substrate wettability also significantly improves the quality of graphoepitaxial layers [24] (Figure 4(b)).

In both cases, the height of the crystallization medium (melt or solution) decreases in the initial stage due to the capillary forces. This effect impedes growth of epitaxial nuclei in the direction normal to the substrate surface and facilitates their growth in the tangential direction. As a result, the substrate orienting role increases and a transition to the layer-by-layer growth mechanism occurs with a decrease in the growth step height. Consequently, the minimum height of the continuous film decreases and the 

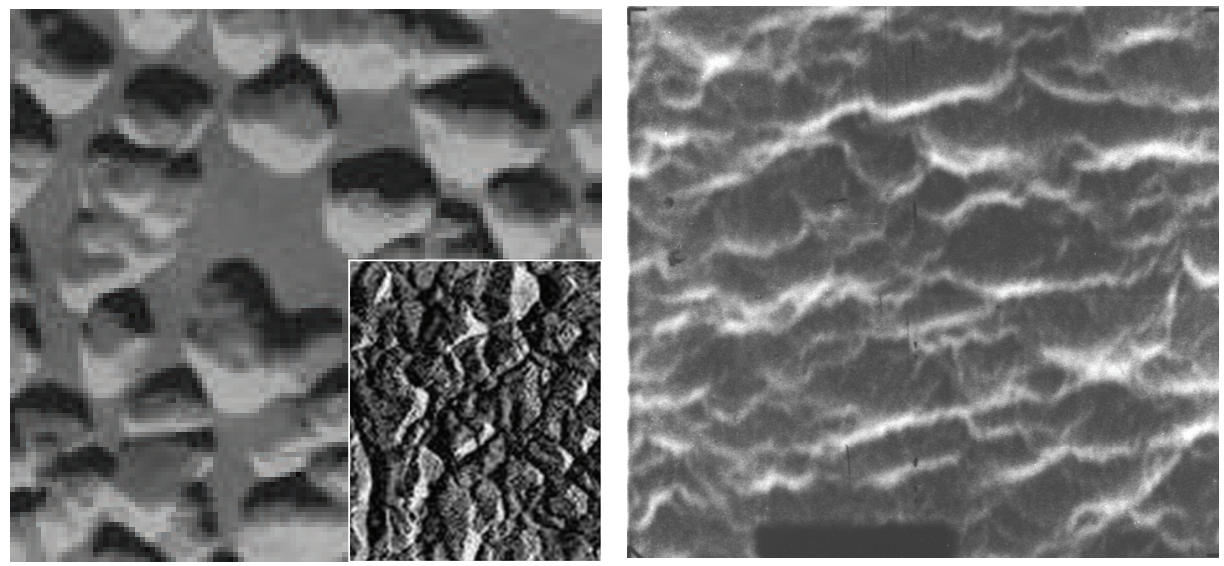

(a)
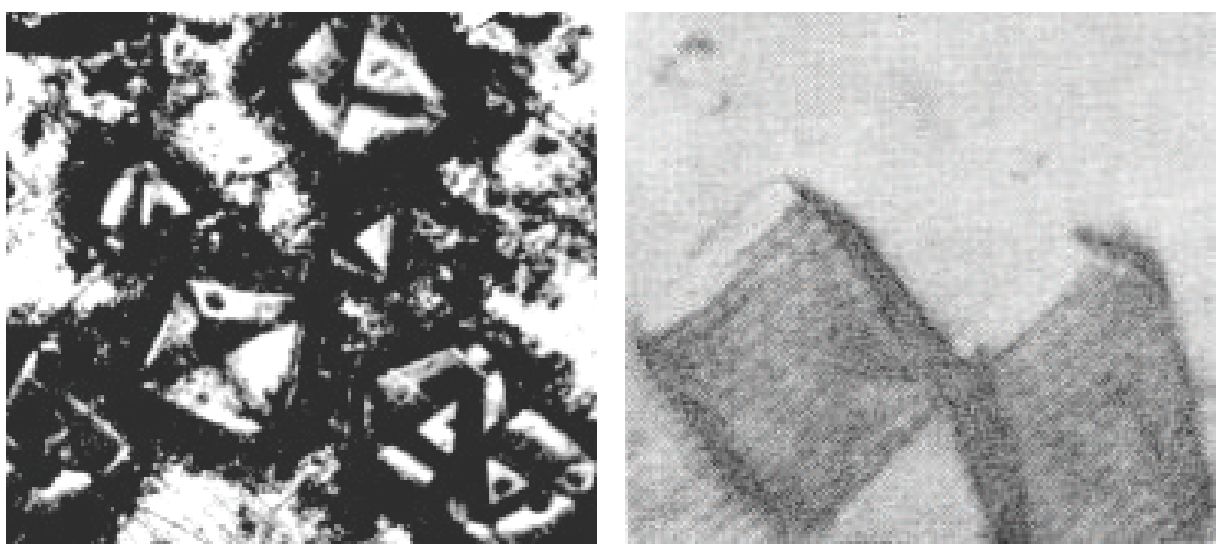

(b)

FIgure 4: Analogy between the capillary epitaxy and graphoepitaxy. (a) Electron microscopy image of GaAs on YSZ at the initial stage of growth (20000x): conventional MOCVD, height of the islets is up to $3000 \mathrm{~nm}$. (b) The capillary epitaxy technique, minimal layer thickness is $50 \mathrm{~nm}$, the layer growth is visible [18]. Optical microscopy image of $\mathrm{NH}_{4} \mathrm{~J}$ on amorphous $\mathrm{Al}$ graphoepitaxy growth: without (c) and with (d) the use of surface-active substances, magnification 100x [24].

film structural quality is improved. It has been shown that the use of capillary forces in this technique improved both the mechanism of epitaxial growth and quality of $\mathrm{A}^{\mathrm{III}} \mathrm{B}^{\mathrm{V}}$ epitaxial films. It also reduces the minimum thickness of a continuous layer $[14,18]$. Virtually the same approach to deposition of $\mathrm{A}^{\mathrm{III}} \mathrm{N}$ films on various substrates has been successfully applied in studies of the other authors [26].

The use of capillary epitaxy made it possible to decrease minimum thickness of a continuous GaAs/fianite film to $25 \mathrm{~nm}$ and to improve its structural quality and surface morphology. The technique was also efficient for growing of the other $\mathrm{A}^{\mathrm{III}} \mathrm{B}^{\mathrm{V}}$ compounds on fianite.

2.4.3. Deposition of GaAs, AlGaAs, and InGaAs-Based Multilayer Structures on Fianite. The results on epitaxial growth of $\mathrm{A}^{\mathrm{III}} \mathrm{B}^{\mathrm{V}}$ compound films obtained in the studies described above were used for obtaining of $\mathrm{AlGaAs} / \mathrm{InGaAs} / \mathrm{GaAs}$ multilayer heterostructures on fianite. These structures were used in FET. Sequential growth $\mathrm{A}^{\mathrm{III}} \mathrm{B}^{\mathrm{V}}$ heteroepitaxial layers on fianite substrates was conducted according to the topologic scheme of PHEMT (Pseudomorphic High Electron
Mobility Transistor) for microwave frequency FET operating in 10-40 GHz range (Table 2) using "Aixtron AIX 200RF" installation. Capillary epitaxy MOCVD technique in 550$600^{\circ} \mathrm{C}$ temperature range was used.

Grown by the "capillary epitaxy" technique series of GaSb and GaAs:Sb buffer layers on (111) and (100) fianite substrates were developed to decrease surface roughness of the PHEMT heterostructure. The buffer layers had a uniform mirror-smooth surface of about $5 \mathrm{~nm}$ roughness. Application of the developed buffers made it possible to obtain an $\mathrm{AlGaAs} / \mathrm{InGaAs} / \mathrm{GaAs}$ heterostructures with uniform mirror-smooth surface on fianite substrates and to decrease its roughness by a factor of 10 (to $25 \mathrm{~nm}$ ). As a result, sufficiently homogeneous $\mathrm{AlGaAs} / \mathrm{InGaAs} / \mathrm{GaAs}$ multilayer heterostructures with smooth slightly bloom surface were grown on (001) fianite substrates of $50 \mathrm{~mm}$ diameter. Roughness of the heterostructure surface measured using Talysurf interference microscope (3-dimensional topography) was $0.25 \mu \mathrm{m}$. This structure was grown using "AIXTRON" installation on (100) fianite ellipsoidal substrate of 2 inch major diameter. The surface of the multilayer 
TABLE 2: PHEMT heterostructure for FET operating in $10-40 \mathrm{GHz}$ range.

\begin{tabular}{|c|c|c|}
\hline$n^{+} \mathrm{GaAs}: \mathrm{Si}$ & $n_{\mathrm{Si}} \sim 6 \times 10^{18} \mathrm{~cm}^{-3}$ & $40 \mathrm{~nm}$ \\
\hline $\mathrm{i}-\mathrm{Al}_{x} \mathrm{Ga}_{1-x} \mathrm{As}$ & $x \sim 0.24(>0.23)$ & $25 \mathrm{~nm}$ \\
\hline i-GaAs & & $\sim 0.6 \mathrm{~nm}$ \\
\hline$\delta-\mathrm{Si}$ & $n_{\mathrm{Si}} \sim 4.5 \times 10^{12} \mathrm{~cm}^{-2}$ & \\
\hline $\mathrm{i}-\mathrm{GaAs}$ & & $\sim 0.6 \mathrm{~nm}$ \\
\hline $\mathrm{i}-\mathrm{Al}_{x} \mathrm{Ga}_{1-x} \mathrm{As}$ & $x \sim 0.24$ & $4 \mathrm{~nm}$ \\
\hline $\mathrm{i}-\mathrm{GaAs}$ & & $1 \mathrm{~nm}$ \\
\hline $\mathrm{i}-\mathrm{In}_{y} \mathrm{Ga}_{1-y} \mathrm{As}$ & $y \sim 0.18(<0.2)$ & $11 \mathrm{~nm}$ \\
\hline i-GaAs & & $30 \mathrm{~nm}$ \\
\hline $\mathrm{i}-\mathrm{Al}_{x} \mathrm{Ga}_{1-x} \mathrm{As}$ & $x \sim 0.24$ & $50 \mathrm{~nm}$ \\
\hline i-GaAs & $n<8 \times 10^{14} \mathrm{~cm}^{-3}$ & $0.5-0.8 \mu \mathrm{m}$ \\
\hline CP AlAs/GaAs & & $(1 \mathrm{~nm} / 2 \mathrm{~nm}) \times 5$ \\
\hline \multirow[t]{2}{*}{ GaAs:Sb } & & $100 \mathrm{~nm}$ \\
\hline & Fianite substrate & $400 \mu \mathrm{m}$ \\
\hline
\end{tabular}

structure is rather uniform but its roughness reaches the value of $25 \mathrm{~nm}$.

Structural perfection of AlGaAs/InGaAs/GaAs multilayer heterostructures on fianite was investigated by means of XRD. DRON-4 device (Ge(004) monochromator, $\mathrm{CuK} \alpha 1$ radiation) was used. $\Theta / 2 \Theta$ spectra were recorded at symmetric reflection mode by scanning with 0.1 step of the texture maxima rocking. X-ray diffraction $\Theta / 2 \Theta$ spectrum of GaAs (001)/fianite (001) is shown in Figure 5. The peaks of $(\mathrm{Zr}, \mathrm{Y}) \mathrm{O}_{2}(004), 2 \theta=73.4$ substrate and of GaAs (004), $2 \theta=$ $66.05^{\circ}$ buffer layer were recorded. The width of the layer rocking curve $\mathrm{FWHM}_{\omega}=1$, that is, the evidence of a mosaic structure of GaAs layer. The grain-boundary angle was $\sim 1^{\circ}$. The use of (111) fianite substrates with GaAs:Sb buffer layers for deposition of $\mathrm{AlGaAs} / \mathrm{InGaAs} / \mathrm{GaAs}$ heterostructures on fianite resulted in the formation of mirror-flat homogeneous surface and 10-fold decrease of the surface roughness (up to $0.025 \mu \mathrm{m})$.

Detailed data on elemental and molecular compositions of the heterostructures were obtained by means of layer-by-layer SIMS ("TOF SIMS-5" spectrometer). The sputtering was carried out by $\mathrm{Cs}^{+}, 2 \mathrm{keV}$, raster $250 \times$ $250 \mu \mathrm{m}$, negative ion detection mode, the probe beam $\mathrm{Bi}^{+}$, $25 \mathrm{keV}$, and depth resolution $\mathrm{DZ}>7 \mathrm{~nm}$. The analysis of the $\mathrm{AlGaAs} / \mathrm{InGaAs} / \mathrm{GaAs}$ heterostructures obtained on fianite (Figure 5(b)) has shown that its inner topology was in conformity with the assigned scheme (Table 2) of the PHEMT structure.

2.4.4. $A^{I I I} N$ Films on Fianite Substrates and Buffer Layers. Principal difficulty of growth of perfect heteroepitaxial GaN films is an absence of suitable substrates having good matching with the heteroepitaxial film. Currently, for the growth of GaN films, $\mathrm{Al}_{2} \mathrm{O}_{3}, \mathrm{ZnO}, \mathrm{MgO}, \mathrm{SiC}, \mathrm{Si}$, and $\mathrm{GaAs}$ substrates are in use. Usually, a material with wurtzite structure is grown on a hexagonal substrate, whereas sphalerite is grown on a cubic one. Fianite as a substrate material for cubic InGaN epitaxy has a number of advantages, such as favorite crystallochemical parameters and high chemical stability. Besides fianite, Si and GaAs substrates with fianite buffer layer were developed in scope of the work. Synthesis of the layer was carried out by a laser deposition technique. The growth of fianite films on silicon substrates was conducted with the purpose to evaluate prospects of the use of less expensive large silicon substrates with fianite sublayer instead of monolithic fianite because maximum dimensions of the silicon-on-fianite structures are limited by size and quality of fianite crystals and the corresponding substrates (currently $\sim 50 \mathrm{~mm}$ ).

Another purpose of the study was determination of suitability of fianite not only as a substrate material but also as a gate dielectric. Production of such substrates will allow integrating GaN-based optoelectronics with a well-developed silicon and gallium arsenide electronics and optoelectronics.

GaN Films on Fianite Substrates. Growth of the films on (111) and (100) oriented fianite substrates was carried out using nucleus layers. 3 types of the nucleus layers were used:

(1) low-temperature GaN nucleus layer with annealing in hydrogen-ammonia atmosphere;

(2) low-temperature AlN nucleus layer with annealing in hydrogen-ammonia atmosphere;

(3) high-temperature AlN nucleus layer.

At the use of all of the types of the nucleus layers, fianite substrates were annealed in pure hydrogen at $\sim 1070^{\circ} \mathrm{C}$ before deposition of the films.

Hydrogen is a conventional carrier gas in MOGPE of III-V materials because it can be rather readily purified. Similarly, in MOGPE of nitrides of III group hydrogen for the first time was used as a carrier gas. However, later it was demonstrated that in contrast to classic III-V semiconductors, GaN and InN are unstable under hydrogen atmosphere and undergo destruction (etching) at the temperatures used for growth of these crystals. This is an evidence for hydrogen as a carrier gas at the epitaxy of nitrides of III group elements actively participates in the process occurring on the surface of the growing film, in contrast to GaAs. Therefore, in most cases for growth of nitrides of III group by MOGPE, ammonia is used as a nitrogen source and supplied into reactor in large quantities. For a long time, ammonium was of opinion that it inhibits the destruction of a growing film and makes the effect of hydrogen negligible. However, it appears that it is far from the case and hydrogen significantly influences the process of the nitrides growth.

The studies have shown that at annealing of LT-GaN nucleus layer, the latter undergo etching in $\mathrm{H}_{2}-\mathrm{NH}_{3}$ flow hindering growth of a high-quality GaN films. Application of the low-temperature AlN nucleus layer with annealing in hydrogen-ammonia atmosphere as well as the hightemperature AlN nucleus layer on (111) and (100) oriented fianite substrates resulted in formation of hexagonal GaN films comprising a textured polycrystal of hexagonal modification. Scattering angles of the texture for the GaN films 


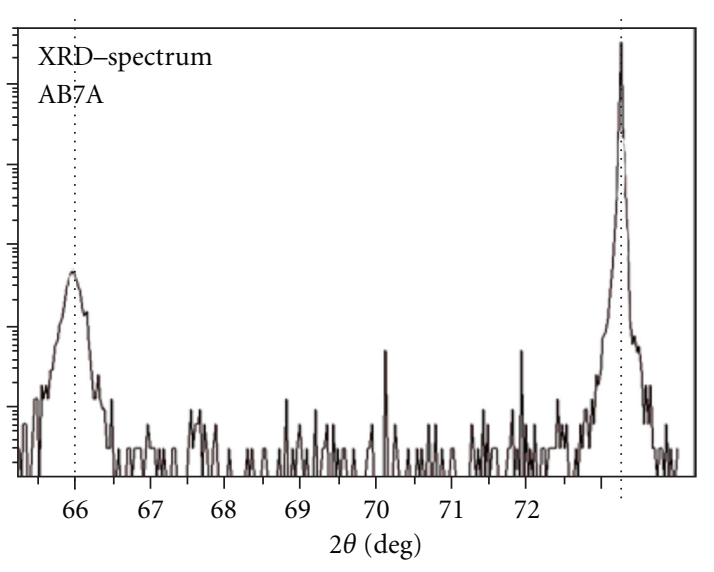

(a)

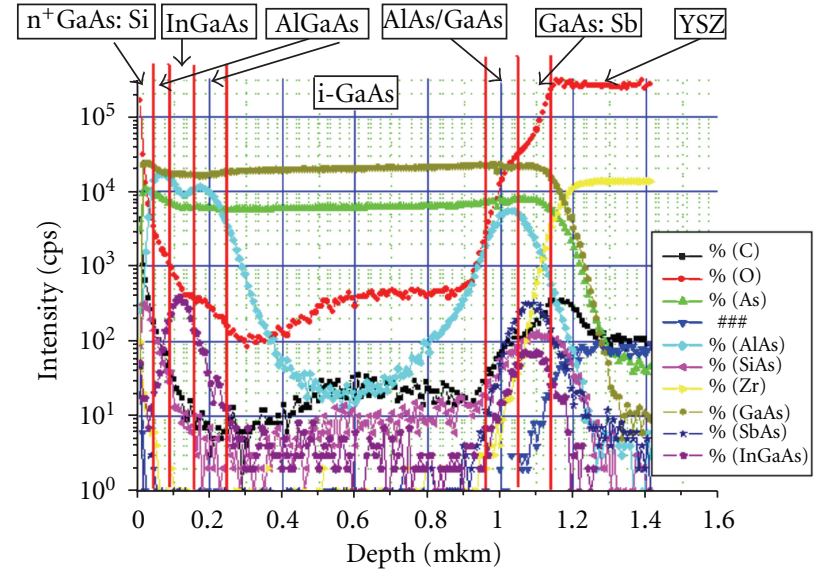

(b)

FIGURE 5: XRD $\theta / 2 \theta$ scan (a) and layer-by-layer secondary ion mass-spectrometry (b) of AlGaAs/InGaAs/GaAs (001)/fianite (001) multilayer heterostructure.

grown on the (111) and (100) oriented substrates were $10^{\circ}$ and $15^{\circ}$, respectively.

It has been shown that high-temperature annealing of LT-GaN buffer layer at $1000-1100^{\circ} \mathrm{C}$ promotes improvement of structural perfection $\mathrm{GaN}$ heteroepitaxial films. The GaN layers on fianite substrates exhibited an intense photoluminescence with maximum at $365 \mathrm{~nm}$.

The conditions of growth of single-crystal GaN films on (111) and (100) fianite substrates by MOCVD without buffer layer at $850^{\circ} \mathrm{C}$ substrate temperature have been determined. The spectra of $\theta / 2 \theta$ scanning were obtained using Ge (400) monochromator (Figure 6).

Two peaks of the substrate were observed at $30^{\circ} \mathrm{YSZ}$ (111) and $34.8^{\circ}$ YSZ (200). The film provides a single GaN (0002) peak at $34.5^{\circ}$. Since GaN (0002) peak is close to YSZ (200) one, a narrow slit in front of the detector was inserted with the purpose to increase the resolution. $\mathrm{GaN}_{\text {hex }}$ (0001) was detected on both substrates at FWHM of the $\mathrm{XRC}<1^{\circ}$ that corresponds to the epitaxial growth. Traces of the polycrystalline phase at $32.4^{\circ}$ (expected $0.1-1.0$ intensity units) were not detected.

AlN Films on Fianite Substrates. The AlN films on fianite substrates were grown using an MOGPE technique. The $\mathrm{Al}_{x} \mathrm{Ga}_{1-x} \mathrm{~N}$ direct gap semiconductors are very useful in the development of UV photodetectors. By altering $\mathrm{Al}$ content in GaN-based solid solutions, it is possible to obtain the material with a forbidden band ranging 3.43-6.2 eV thus covering $200-365 \mathrm{~nm}$ spectral band. This spectral band is of practical importance in UV astronomy, ozone layer monitoring, combustion, and water sensors. These films are both of original interest as well as are useful as nucleating and buffer layers in GaN epitaxy.

Growth of the films was started from thin $20-50 \mathrm{~nm}$ nucleating layer. Two growth modes were used: at $650^{\circ} \mathrm{C}$ with subsequent annealing in ammonia-hydrogen media at $1100^{\circ} \mathrm{C}$ during $30 \mathrm{~min}$ followed by growing up of the basic layer and high-temperature growth of AlN at the same

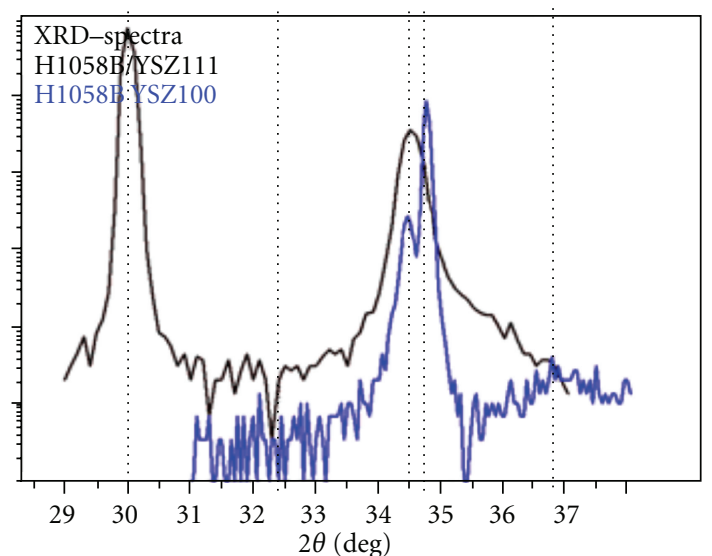

Figure 6: XRD $\theta / 2 \theta$ scan of GaN films on (111) and (100) fianite substrates.

temperature. Before the deposition of AlN films, fianite substrates were annealed in pure hydrogen at $\sim 1070^{\circ} \mathrm{C}$. Mirror-flat homogeneous AlN films with the roughness not exceeding $0.6 \mathrm{~nm}$ (Figure 7) were deposited on (100) and (111) fianite substrates.

Layer-by-layer analysis of AlN nucleating layer on the fianite substrates was carried out by SIMS using TOF SIMS-5 device (sputtering by $\mathrm{Cs}+, 2 \mathrm{keV}, 250 \times 250$ raster, negative recording mode, $\mathrm{Bi}+$ probe beam $25 \mathrm{keV}$ ).

The study has shown that the films had uniform distribution of its constituents, the concentration profile of $\mathrm{Zr}$ atoms at the heterointerface being very sharp (Figure $7(\mathrm{c})$ ). The use of AlN nucleating layers on the fianite buffering layers allows deposition of continuous and homogeneous GaN films of hexagonal modification.

Deposition of InN Single-Crystal Films on YSZ Substrates by Means of LP-MOCVD Technique Using Pulse-Capillary Epitaxy. InN heteroepitaxial films attract a significant interest 


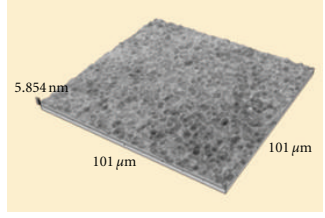

(a)

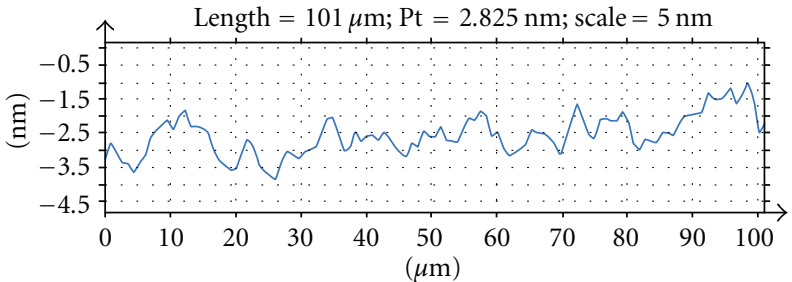

(b)

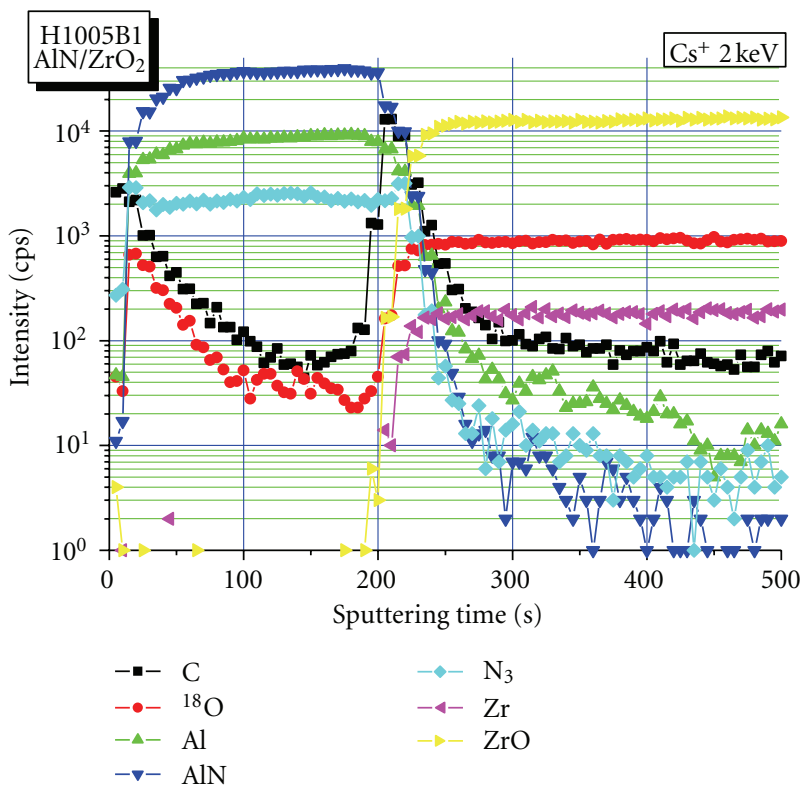

(c)

FIGURE 7: Image of surface (a) and the surface relief (b) (Interference Microscope “Talysurf”); results obtained by layer-by-layer SIMS analysis (c) of the low temperature AlN layer on fianite substrate (TOF SIMS-5).

because the films provide an opportunity of the development of high-frequency electronic devices. Since an industrial technology of InN substrates production is so far absent, heteroepitaxial growth of $\mathrm{InN}$ on foreign substrates is required. $\mathrm{Al}_{2} \mathrm{O}_{3}$ and Si substrates, being well developed and having considerable dimensions along with excellent crystal perfection, seem to be of the most interest. Moreover, Si is not expensive and allows a combination of the InN-based devices with well-developed silicon electronics. However, mismatching between $\mathrm{Al}_{2} \mathrm{O}_{3}, \mathrm{Si}$, and InN lattice constants is rather large, $25 \%$ and $8 \%$, respectively. That was the reason why fianite (YSZ) has been recently taken into account as an alternative substrate.

For the first time fianite substrates have been successfully tested for deposition of $\mathrm{InN}$ heteroepitaxial films in [4]. The films of cubic symmetry were grown on (001) fianite substrates using plasma-assisted molecular beam epitaxy (PA-MBE) at $400-490^{\circ} \mathrm{C}$.

Due to better crystal-chemical matching between InN and fianite, the films grown on fianite substrates exhibited crystal perfection better than those grown on sapphire and GaAs substrates [4].
In [5], InN epitaxial films have been for the first time deposited on fianite substrates by pulse-capillary LPMOCVD. The technique was shown to allow a significant improvement of the films quality, as well as synthesizing single-crystal InN films of hexagonal modification. The films demonstrated rather perfect morphology along with high photoluminescent and electrophysical characteristics. The structures on fianite substrates were grown under low pressure in vertical reactor supplied with rotating molybdenum stand (designed in IPM RAS). The substrates were inductively heated, trimethyl indium (TMIn) and ammonia were the element sources, and nitrogen was the carrier gas. The substrates of (111) and (100) orientations were used. Comparative studies of the InN films were carried out using optical and electron microscopy, SIMS, XRD, photoluminescence, and probe techniques.

The experiments on deposition of InN films on fianite by MOCVD technique have shown that it was difficult to grow a uniform and smooth $\mathrm{InN}$ film using general growth process, as well as single-step capillary epitaxy, because of three-dimensional nucleation. Satisfactory results have been achieved only by using pulse-capillary epitaxy, in which the process of intermittent supply of In (in form TMIn) at 


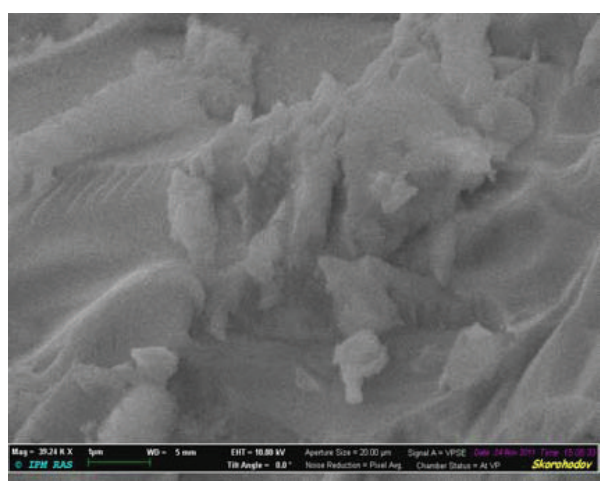

(a)

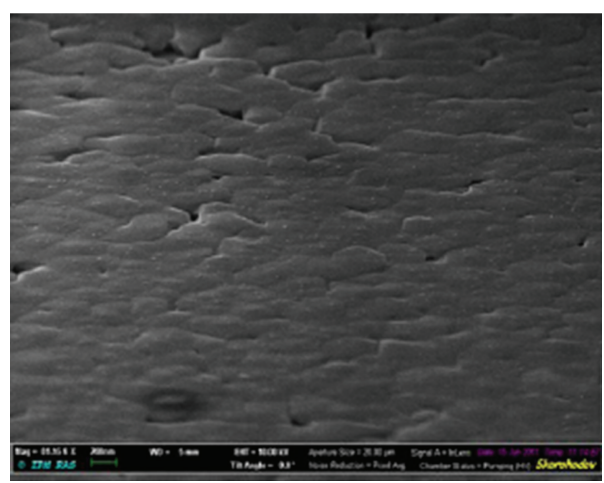

(b)

FIGURE 8: Morphology of the surface of InN films deposited by means of conventional (a) and pulse-capillary LP-MOCVD techniques (b) (scanning electron microscope Supra 50 VP).

constant $\mathrm{NH}_{3}$ flow was repeatable [7]. The pulse-capillary LP-MOCVD deposition was started by puffing TMIn in nitrogen without ammonia inflow during $20 \mathrm{~s}$ followed by $40 \mathrm{~s}$ interruption of TMIn supply and puffing nitrogenammonia mixture. TMIn flow was equal to $0.25 \mathrm{sccm} / \mathrm{min}$. The procedure was repeated ten times and then the growth

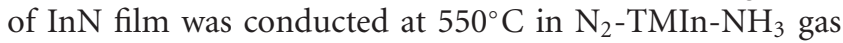
mixture. The V/III reactant ratio was in the range of $10^{5}-10^{6}$. Under these conditions growth of InN films of $0.3 \mu$ thickness was observed. The application of "pulse-capillary epitaxy" technique and fianite substrates resulted in the formation of mirror-flat films of single-crystal structure. The comparative studies on growth (nucleation and formation of the primary continuous layer) of InN films on fianite substrates by means of LP-MOCVD and conventional MOGPE techniques have demonstrated significant differences in size, habitus, and concentration of the epitaxial nuclei, as well as in morphology of the epitaxial layers. Raster electron microscopy (Supra $50 \mathrm{VP}$ ) images of the surface of InN layers deposited by means of conventional LP-MOCVD (a) and pulsecapillary LP-MOCVD techniques (b) are shown in Figure 8. Roughness of the surface of InN layer of $0.3 \mu$ thickness grown by the conventional technique is apparently seen. The roughness occurred because of the three-dimensional nucleation.

At the same time, pulse-capillary epitaxy resulted in more smooth and uniform surface. $\theta / 2 \theta$ scanning spectrum of InN film deposited on (111) fianite substrate by pulse-capillary LP-MOCVD technique and rocking curves of InN films are presented in Figure 9. The $2 \theta$ peaks in the spectrum are attributed to 30.0-YSZ (111) substrate, 31.3-InN (0002) layer, and 33.0- In (100).

The rocking curve of InN (0002) film deposited on YSZ substrate by means of pulse-capillary epitaxy is shown in Figure 10. Rocking curves of InN films deposited on YSZ and sapphire substrates by means of conventional techniques are shown for comparison. The rocking curve data of $\mathrm{InN}$ (0002) peak provide full width at half maximum $1,8^{\circ}$ for the films grown on sapphire (curve 3 on Figure 10). The values obtained for fianite films grown using conventional

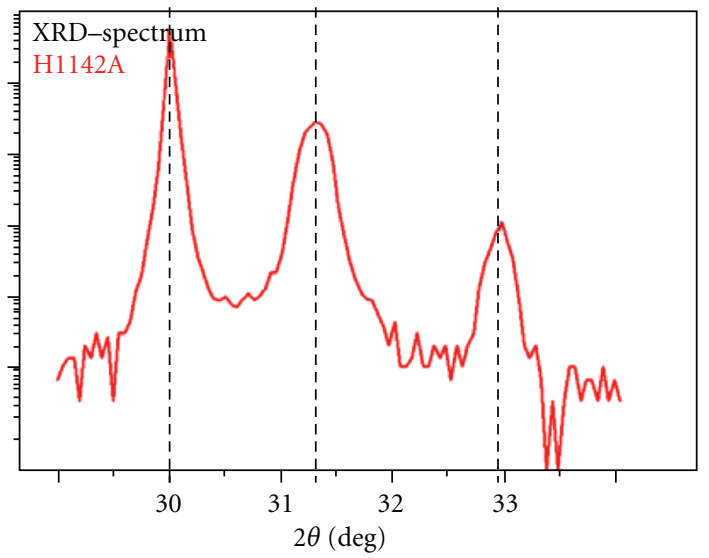

Figure 9: XRD $\theta / 2 \theta$ scan of the InN film.

and LP-MOCVD using pulse-capillary epitaxy are $1.0^{\circ}$ and $0.19^{\circ}$ (curves 2 and 1 on Figure 10), respectively. It is noticeable that the latter value is considerably lower. Therefore, the growth on fianite results in more structurally perfect InN films due to better crystallographic matching, other conditions being equal. Herewith, application of the pulse-capillary epitaxy allows achieving of record values that is the evidence of excellent perfection of InN films obtained in this study.

The nondoped epitaxial InN films grown on fianite substrates were semiconductors of $n$-type with $2.5 * 10^{19} \mathrm{~cm}^{3}$ electron concentration. It is also noticeable that Hall mobility value approaches $320 \mathrm{~cm}^{2} / \mathrm{V} \times \mathrm{s}$. The films showed intense photoluminescence with peak maximum at $0.87 \mathrm{eV}$.

\subsubsection{Electrically Active Defects in GaN Films on GaAs} Substrates with Fianite Buffer Layers. Comparative studies of density and electric activity of structural defects in $\mathrm{GaN}$ epitaxial films grown on GaAs substrates with various buffer layers were carried out by an induced bias technique (IBT). The technique has been developed rather recently $[27,28]$. It is a contact-free similarity of the induced current 


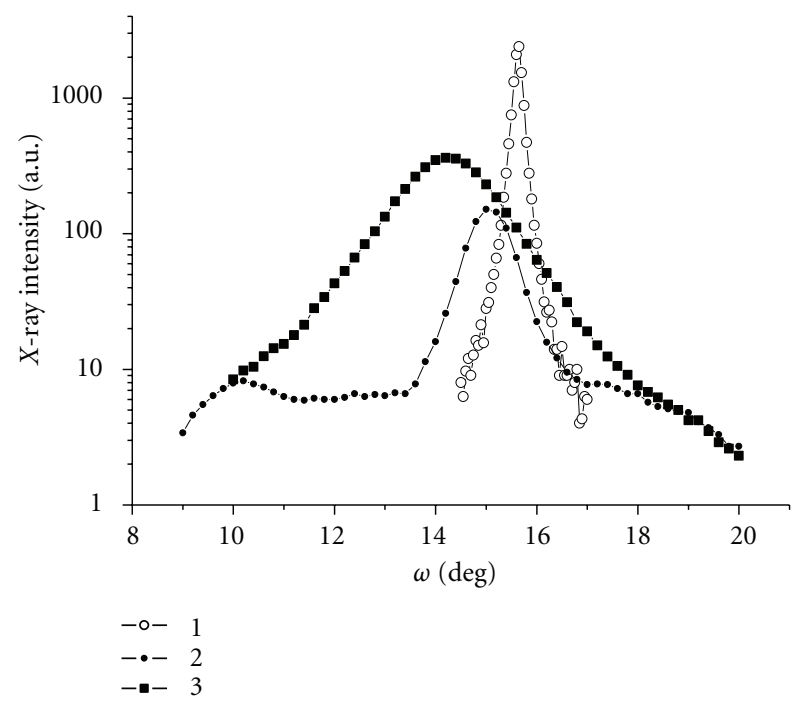

FIGURE 10: Rocking curves of (0002) InN.

technique (EBIC-mode). IBT is a nondestructive contactfree method for diagnostics of semiconducting materials and microelectronic devices. IBT is based on detecting voltage (or charge) generated by an electron probe of scanning electron microscope (SEM). Draft scheme of the method is shown in Figure 11(a).

The electron probe (e) scans the surface of a crystal under the study $(\mathrm{O})$. Metal ring (D), in which the surface charge generated by electrons through capacitive coupling is induced, is a detector of a signal. The signal from the ring electrode is monitored in the SEM display (or by other measurement equipment) through charge-sensitive amplifier (PA) (Figure 11(a)). The technique allows qualitative monitoring of semiconductor plates, structures, and devices identifying electric active inhomogeneities such as dislocations, stacking faults, microfractures, extent of doping by various dopants, all $p$ - $n$ junctions, and Schottky barriers (see e.g., Figures 11(b) and 11(c)). Quantitative measurements of local fundamental characteristics of semiconductors are also possible (diffusion distance, nonequilibrium carrier lifetime, its surface recombination rate, diffusion barrier height).

The studies have shown that the use of GaAs substrates with porous GaAs layer resulted in a decrease of the electric activity of structural defects in the GaN films and in an increase of its electrical uniformity as compared to GaN films grown on monolithic GaAs substrates. The use of GaAs substrates with double buffer layer (fianite on porous GaAs) allows additionally decreasing concentration of the electrically active defects in GaN films to more than an order of magnitude (Figure 12).

2.4.6. GaN Films on Si and GaAs Substrates with Fianite Buffer Layers. Silicon and gallium arsenide are promising substrates for $\mathrm{GaN}$ and the other $\mathrm{A}^{\mathrm{III}} \mathrm{N}$ epitaxy due to their high quality, large dimensions, and a low net cost, as well as possibility to integrate GaN-based devices with well-developed silicon and gallium arsenide electronics and optoelectronics. However, there are three considerable problems occurring at GaN epitaxy: first, a significant parameter mismatch of GaN layer and Si or GaAs substrates; second, the difference of thermal expansion coefficients of the layer and the substrates and third, insufficient chemical and thermal stability of the substrates at the epitaxial temperature. Application of various buffer layers, in particular, fianitebased, can be an efficient method for solution of the above problems.

GaN epitaxial films were grown by MOCVD technique using capillary epitaxy on $\mathrm{Si}$ and GaAs substrates with various buffer layers. Trimethylgallium (TMG), arsine $\left(\mathrm{AsH}_{3}\right)$, and ammonia $\left(\mathrm{NH}_{3}\right)$ were used as $\mathrm{Ga}, \mathrm{As}$, and $\mathrm{N}$ sources, respectively. Single (fianite, layer of porous $\mathrm{Si}$, or GaAs material) and double (fianite on porous Si and GaAs) were tested. The first "prominent" porous buffer layer was suggested to allow decreasing thermoelastic strains in the second heteroepitaxial buffer thus improving its morphology and structure. The upper buffer layer, being chemically stable in the growth medium, provides fine matching with functional heteroepitaxial film.

The epitaxial structures were studied using a set of techniques: photoluminescence (PL), scanning electron microscopy in electron-beam induced current (EBIC-mode), and secondary-ion mass spectroscopy (SIMS).

It was established that the use of fianite buffer layer on Si substrate prevents formation of amorphous silicon nitride. The GaN films grown on Si substrates with fianite buffer layer were of hexagonal modification $(\alpha-\mathrm{GaN})$ and had mosaic single-crystal structure. It was demonstrated that the use of porous Si in the complex fianite/Si buffer allows improving the adhesion of GaN film and its uniformity by phase composition and thickness.

Layer-by-layer SIMS analysis of GaN films grown on $\mathrm{Si}$ and GaAs substrates with fianite buffer layers has shown that fianite layer serves as a barrier for diffusion of $\mathrm{Si}$ and As into GaN film from Si and GaAs substrates, respectively (Figure 13). Good insulating properties of $\mathrm{ZrO}_{2}$ in the double buffer provide an opportunity to use "Semiconductor on dielectric" technology, which is promising to improve the integration level.

Comparative studies of PL spectra (recorded at $300^{\circ} \mathrm{K}$ ) of GaN films grown on a monolithic GaAs substrate and GaAs substrates with various kinds of buffer layers have been carried out (Figure 14): 1 single buffer "porous GaAs;" 2 double buffer "fianite on porous GaAs."

The position of PL peaks in the spectra corresponds to characteristic peak of cubic GaN. Consequently, the use of single buffer layer of porous GaAs as well as double buffer layer (fianite on porous GaAs) allows growing GaN films of cubic modification. The growth of GaN film on monolithic GaAs substrate in contrast resulted to the formation of hexagonal modification.

\section{Functional Fianite Films}

3.1. Functional Fianite Films on Si, Ge, and GaAs Substrates. Thin films of fianite and related solid solutions such 


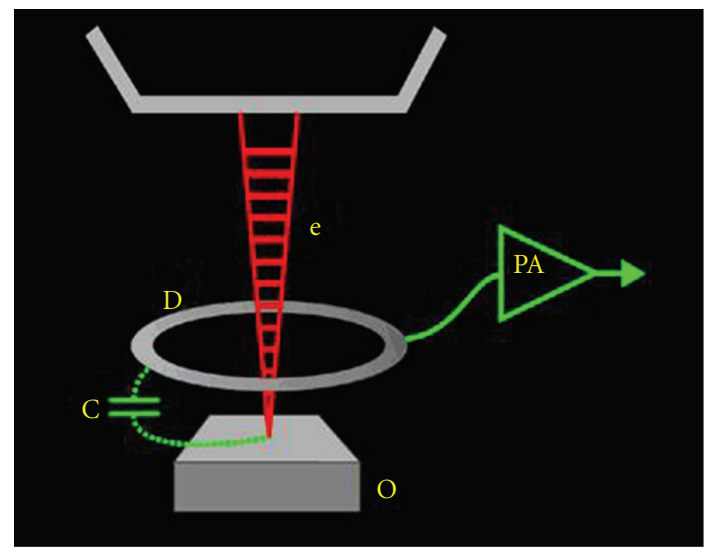

(a)

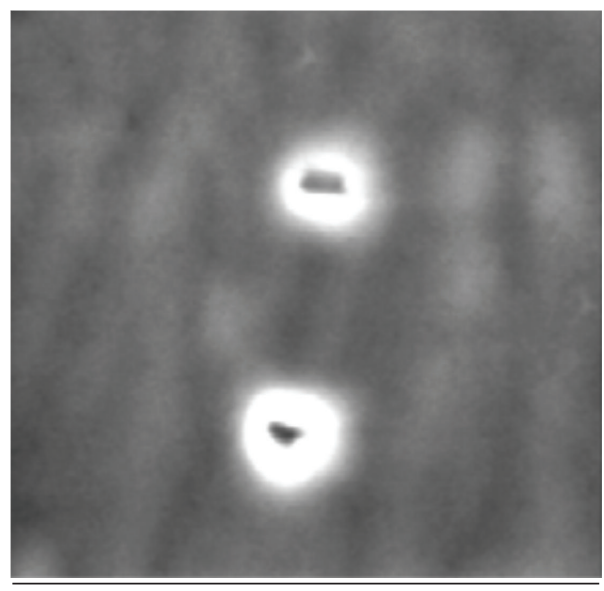

$20 \mathrm{kV} \times 10000 \overline{1 \mu}$

(b)

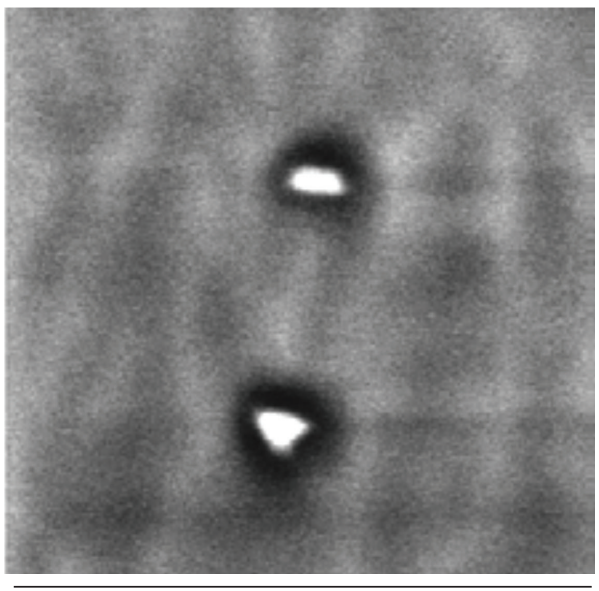

$20 \mathrm{kV} \times 10000 \overline{1 \mu}$

(c)

FIGURE 11: Outline of the induced potential technique (a) and scanning electron microscope images of electrically active polygonal defects in GaAs films: secondary-electron emission mode (b); b-induced potential mode (c).

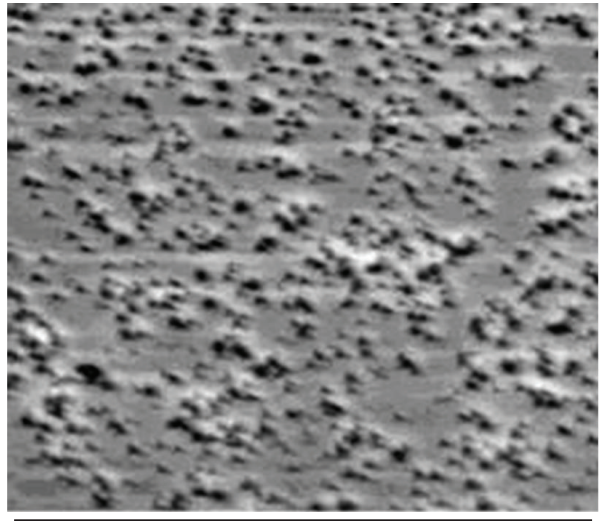

$20 \mathrm{kV}$ x1000 $\overline{10 \mu}$

(a)

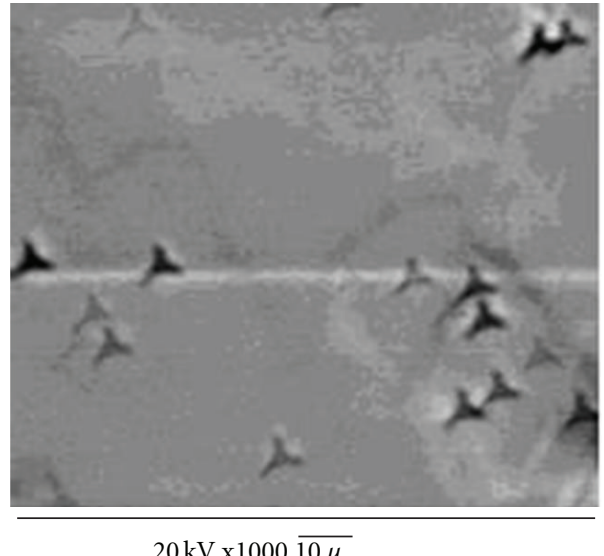

(b)

Figure 12: Electrically active defects in GaN film on GaAs substrate with buffer layers: (a) single buffer (fianite); (b) double buffer (fianite on porous $\mathrm{GaAs}$ ). 


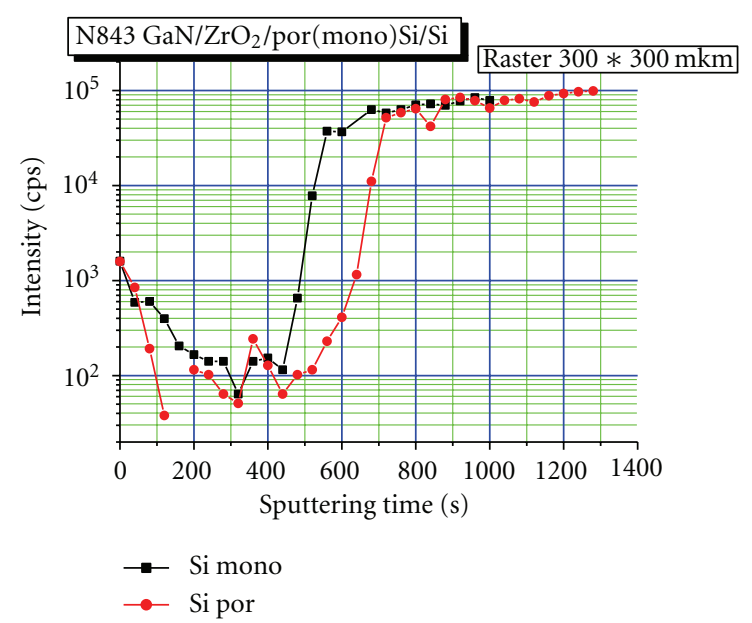

(a)

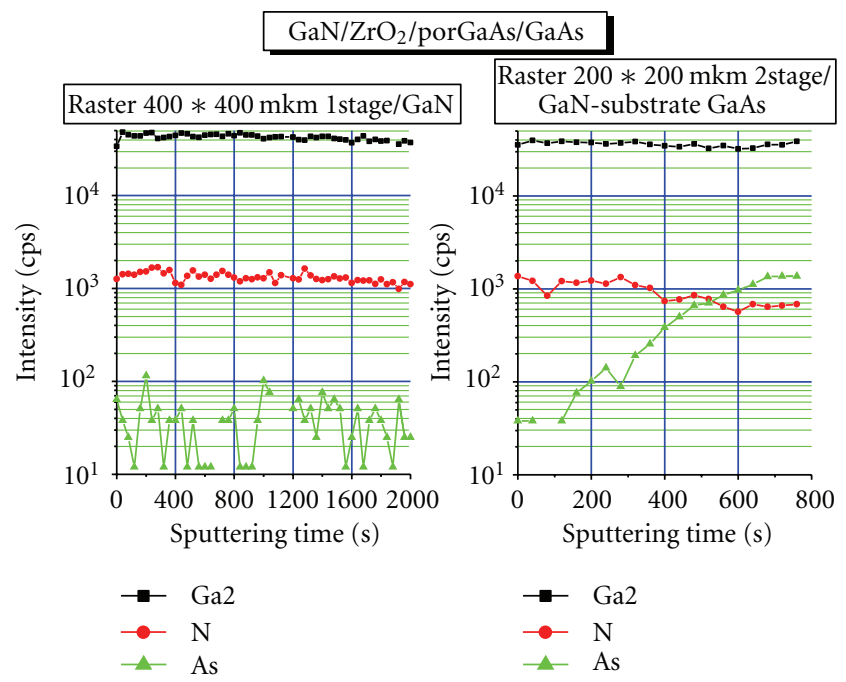

(b)

FIGURE 13: Results from a layer-by-lyer SIMS analysis of GaN/ fianite/por(mono)Si/Si (a) and GaN/fianite/porGaAs/GaAs (b) structures.

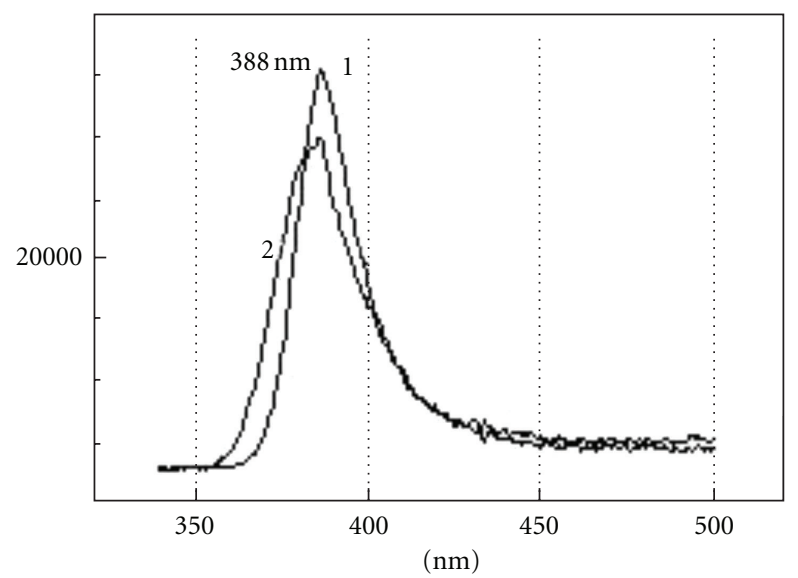

FIGURe 14: Photoluminescence spectra of GaN films $\left(300^{\circ} \mathrm{K}\right)$ on GaAs substrate with buffer layers: porous GaAs (1) and double buffer-fianite on porous GaAs (2). as $\mathrm{Zr}(\mathrm{Ce}) \mathrm{O}_{2}$ can be used as insulating layers (alternative to $\mathrm{SiO}_{2}, \mathrm{SiC}$, and $\mathrm{Si}_{3} \mathrm{~N}_{4}$ ) in the development of $\mathrm{Si}$-, Ge- and GaAs-based "semiconductor-dielectric" multilayer structures. Fianite is also a good gate dielectric for Si- as well as for $\mathrm{A}^{\mathrm{III}} \mathrm{B}^{\mathrm{V}}$-based devices (including GaN-based) due to its high dielectric constant value (25-29.7). Thin fianite films are a barrier for diffusion of impurities and provide a significant (up to 1000-fold and even more) decrease of the loss current in highly integrated devices $[11,12]$. Due to high chemical inertness fianite films can also be used as protective coatings.

3.2. Techniques for Deposition of Fianite Films on Si and GaAs Substrates. In recent years, a considerable attention was drawn to fianite films on silicon due to its electric and optic device applications, such as insulating layers in SOI (silicon-on-insulator) devices [29], gate dielectric in Si$[28,29]$, SiGe- [30], and $\mathrm{A}^{\mathrm{III}} \mathrm{B}^{\mathrm{V}}$-based [31] device structures, buffer layers for production of optic coatings for films of various semiconductors [30-33], superconductors [34-36], ferroelectrics.

Various techniques can be used for the production of fianite films on silicon and other semiconductors, including magnetron [36-40], laser and electron-beam [41-43] sputtering, and molecular-beam epitaxy (MBE), as well as gasphase chemical deposition [44].

The choice of a specific technique is determined by further designation of a fianite film and possibility to produce the film of maximum structural perfection, as well as technologic potentialities of the technique. So, MBE technique is more suitable for the deposition of the thinnest fianite film for the use as a gate dielectric. Magnetron and laser sputtering are more favorable for fianite layers used as buffer layers with subsequent growing semiconductor films, including $\mathrm{A}^{\mathrm{III}} \mathrm{B}^{\mathrm{V}}$ compounds. In [36], fianite films were deposited on $\mathrm{Si}$ and GaAs substrates using magnetron, laser, and electron-beam sputtering techniques. The films obtained by magnetron sputtering were of the best structural perfection [36].

3.3. Growing of Fianite Films on Si and GaAs Substrates. The growth of fianite films on silicon and gallium arsenide substrates was carried out with the purpose to evaluate the prospects of using less expensive and more large $\mathrm{Si}$ and GaAs substrates with fianite sublayer instead of monolithic fianite substrates because, currently, maximum size of the latter is $\sim 50 \mathrm{~mm}$. Another purpose was the determination of an opportunity to use fianite not only as a substrate but also as insulating layer material alternative to $\mathrm{SiO}_{2}$, $\mathrm{SiC}$, and $\mathrm{Si}_{3} \mathrm{~N}_{4}$ protecting and insulating layers, as well as a gate dielectric for multi-layer "semiconductor-dielectric" structures. Producing such substrates will allow integrating GaN-based optoelectronics with a well-developed silicon and gallium arsenide electronics and optoelectronics. Magnetron and laser sputtering were used for deposition of fianite films on silicon and gallium arsenide 
3.4. Fianite Films on Porous Si and GaAs. With the purpose to improve quality of fianite films and its adhesion to Si and GaAs substrates, opportunities of the use of porous layers of the material were studied.

The following results were obtained:

(i) appropriate regimes of deposition of porous GaAs layer on GaAs (111) substrates of $n$ - and $p$-conductivity types were developed;

(ii) appropriate regimes of deposition of the uniform mirror-flat fianite layer on GaAs (111) substrates of $18 \times 18 \mathrm{~mm}$ size were established;

(iii) it has been demonstrated that the use of the porous layers allowed an improvement of adhesion of fianite with GaAs layers;

(iv) the samples of fianite/GaAs and fianite/Si epitaxial substrates have been obtained for subsequent growth of $\mathrm{A}^{\mathrm{III}} \mathrm{N}$ films.

High mechanical and chemical stability of fianite and absence of pores open the prospects of its application as protective and stabilizing coating for various substrates.

3.5. Magnetron Sputtering Technique. Magnetron systems are related to diode-type sputtering systems. The sputtering occurred due to bombardment of a target surface by gas ions (usually Ar) forming in plasma of anomalous glow discharge. Material ions knocked out the target subjected to the bombardment are captured by the magnetic field and maintained complex cycloidal movement by closed trajectory in vicinity of the target surface. High sputtering rate, which is a feature of magnetron systems, is achieved by an increase of the ion current density due to localization of plasma by means of high transverse magnetic field. The increase of sputtering at simultaneous decrease of actuation gas pressure allows a significant decrease of contamination of the films by alien gas impurities. Fianite was grown up on $\mathrm{Si}$ and GaAs substrates using unbalanced magnetron system. Fianite crystals were used as a target. Si substrate subjected to the sputtering was heated by IR radiance. Preparation of the substrates included degreasing, removing of the oxide, and passivating of the surface in ammonium-peroxide solution. Optimization of the conditions of the growth of fianite films on Si substrates was carried out by varying of the sputtering rate, temperature of the substrate, and residual gas pressure.

Bombardment of the target leads to dissociation of zirconium and yttrium oxides to $\mathrm{ZrO}, \mathrm{Zr}, \mathrm{YO}, \mathrm{Y}$, and $\mathrm{O}_{2}$. That is why such parameters as sputtering rate and residual gas pressure considerably influence stoichiometry of the resulting film. Energy of the evaporating particles is rather low $(\sim 0.5-10 \mathrm{eV})$; so for the epitaxial growth of fianite film, a high temperature of Si substrate and optimal rate of the condensate supply are necessary.

The fianite films were studied by means of scanning electron microscopy, ellipsometry, and CV-parameters measurement techniques. The film parameters were found as follows: (i) optic refractive index $n_{d} \sim 2.1 \div 2.2$;

(ii) dielectric constant $\varepsilon \sim 25$;

(iii) absence of defects of porosity type (in $30 \mathrm{~mm}$ diameter sample).

3.6. Laser Sputtering Technique. Experimental installation for deposition of fianite films was a sputtering system composed by a vacuum device and excimer laser. The system has been designed and manufactured in IPM RAS.

Operational oxygen pressure was maintained by vacuum system supplied with a mechanical pump and CHA-2 letting system. Evaporation of the target was performed by LPX200 eximer laser radiation working on $\mathrm{KrF}$ mixture. Wavelength of the radiation was $248 \mathrm{~nm}$, pulse duration $27 \mathrm{~ns}$, the pulse energy $350 \mathrm{MJ}$ (pulse power $1.3 \times 10^{7} \mathrm{~W}$ ), and repetition frequency $50 \mathrm{~Hz}$. Optical system providing a focusing of the laser beam on the target surface consisted of quartz prisms and $30 \mathrm{~cm}$ focal distance lens. The laser beam spot on the target surface was $1 \times 4 \mathrm{~mm}^{2}$. The energy density on the target surface was $\sim 10 \mathrm{~J} / \mathrm{cm}^{2}$. The distance between the target and substrate was $60 \mathrm{~mm}$. Cylindrical targets of $15-20 \mathrm{~mm}$ diameter and $10-30 \mathrm{~mm}$ length were used in the installation. In order to prevent local overheating of the target and to provide a uniform material drift rotation an axial movement of the target was used. Possibility of conducting pre- and postgrowth annealing under oxygen atmosphere at $10 \mathrm{~Pa}-100 \mathrm{kPa}$ pressure and at up to $750^{\circ} \mathrm{C}$ temperature is a peculiarity of the installation.

Ceramic target of $\left(\mathrm{ZrO}_{2}\right)_{1-x}\left(\mathrm{Y}_{2} \mathrm{O}_{3}\right)_{x}$ with $x=0.1$ composition was used for deposition of fianite films. The deposition was carried out on Si and GaAs substrates heated to $600-800^{\circ} \mathrm{C}$ temperature under oxygen atmosphere at approximately $10 \mathrm{~Pa}$. The growth rate of YSZ films was about $0.02 \mathrm{~nm}$ per pulse. Contactless heater of substrates (heating by irradiance) was an original peculiarity of the sputtering system. The heater comprises vertically positioned quartz tube (of $30 \mathrm{~mm}$ inner diameter) supplied with refractory stainless steel heating coil on its outer surface with up to $1 \mathrm{~kW}$ power of the heater. Monitoring and maintenance of the assigned temperature (with $\pm 5^{\circ} \mathrm{C}$ precision) were carried out using precise regulating device and $\mathrm{Pt}-\mathrm{Rh}$ thermocouple positioned under the heating coil. A substrate was fitted in a holder and positioned inside of the quartz tube. Loading of substrates and oxygen supply was maintained through the upper end of the tube.

Technology of growth of dielectric fianite films using the laser sputtering consists of the following stages.

(1) A substrate is loaded to the sputtering system and the vacuum chamber is evacuated up to $\sim 1 \mathrm{~Pa}$ residual pressure.

(2) Letting-to-oxygen is maintained up to the pressure required.

(3) Rotational movement drive of the target is switched on. 
(4) A substrate is heated up to deposition temperature.

(5) The eximer laser (the pulse energy $350 \mathrm{MJ}$, repetition frequency $50 \mathrm{~Hz}$ ) is switched on and the sputtering is started.

(6) Followed by the achievement of the assigned thickness of the film, the laser is switched off.

(7) Followed by the end of the film growth, the chamber is filled with oxygen up to the pressure required.

(8) The structure is annealed.

The substrate heater is switched off and the substrate is cooled to room temperature.

3.7. Initial Stages of Deposition and Structure of Fianite Buffer Layers on Si and GaAs Substrates. The application of fianite as a buffer layer will present a solution route to another very important problem: epitaxy of $\mathrm{A}^{\mathrm{III}} \mathrm{N}$ compounds on $\mathrm{Si}$ and GaAs substrates having large dimensions, high quality, and low net cost.

Single-crystalline heteroepitaxial fianite films of $1000 \AA$ thickness were grown on silicon substrates of up to $50 \mathrm{~mm}$ diameter in vacuum chamber at $p \sim 2 \cdot 10^{2} \mathrm{~Pa}$ pressure, sputtering rate $V_{s} \sim 60 \AA / \mathrm{min}$, and substrate temperature $T_{s} \sim 800^{\circ} \mathrm{C}$.

The studies have shown that the film became continuous as from $100 \AA$ thickness.

$\mathrm{X}$-ray structural studies of $\mathrm{ZrO}_{2}-\mathrm{Y}_{2} \mathrm{O}_{3} / \mathrm{Si}$ structures have shown that the fianite film was single phased and consisted of two layers with different rocking curve values: $0.20^{\circ}$ for the upper layer and $0.96^{\circ}$ for the lower one. Epitaxial relation between the film and the substrate was (100)[100] $\mathrm{Si} / /(100)[100] \mathrm{ZrO}_{2}-\mathrm{Y}_{2} \mathrm{O}_{3}$. The relation was established using diffraction measurements under the following regimes: $\Theta / 2 \Theta$ scanning (simultaneous rotation of the detector and sample over goniometer axis) and $\Psi$ scanning (rotation of the plate in a proper plane at fixed detector position). The former regime was used to determine orientation of the composition plane, the latter-mutual orientation of unit cells of the film, and the substrate in the composition plane.

Spectra of the $\Psi$-scanning of $\left(\mathrm{ZrO}_{2}-\mathrm{Y}_{2} \mathrm{O}_{3}\right) / \mathrm{Si}$ structure for asymmetric (422) reflection of the film (b) and the substrate (a) are shown in Figure 15.

The absence of additional peaks and high peak maximum-to-background ratio $\left(\sim 10^{3}\right)$ are the evidence for $\mathrm{ZrO}_{2}$ $\mathrm{Y}_{2} \mathrm{O}_{3}$ layer is a perfect single-crystal film. The fianite buffer layers grown on $\mathrm{Si}$ and $\mathrm{GaAs}$ were used for $\mathrm{A}^{\mathrm{III}} \mathrm{N}$ compounds epitaxy.

3.8. Some Difficulties in Deposition of Fianite Films on Silicon Substrate. Growth of fianite-on-silicon structures of high quality featuring with sharp interfaces is associated with significant difficulties because of a number of principal problems.

First, silicon surface readily undergoes transformation into $\mathrm{SiO}_{2}$ amorphous layer either due to interaction with oxygen-containing fianite film or oxidative atmosphere usually used at fianite growth. As it has been shown by calculations, fianite should not react with silicon substrate to form $\mathrm{SiO}_{2}$, which has low dielectric constant value, at a direct contact [45]. However, in practice, it is very difficult to avoid the formation of this film at fianite deposition or subsequent high-thermal treatment $[46,47]$. Therefore, a development of special technological tools is necessary. One of the routes to solve the problem has been suggested by the authors [48]. Thin $\mathrm{Zr}$ or Y layer was deposited on Si substrate before fianite deposition. The metals absorb oxygen from $\mathrm{SiO}_{2}$ layer because free energy of both fianite and $\mathrm{Y}_{2} \mathrm{O}_{3}$ formation is lower than of $\mathrm{SiO}_{2}$ one [49]. That leads to a decrease of the layer thickness.

Second, oxygen from fianite layer readily diffuses to silicon substrate or reacts with silicon surface. Secondary phases occurring as a result of the reaction disturb silicon crystal lattice and hinder a perfect growth. Under these circumstances, fianite layers on Si substrates are of amorphous or polycrystalline structure. At the development of gate dielectric technology, these issues are of peculiar importance because thickness of the last layer is about some nanometers.

Therefore, the above data show that the problem of deposition of fianite films on Si substrates is of great interest. The problem of improvement of quality of the layers seems to be very urgent because of a number of principal difficulties occurring due to peculiarities of physical-chemical properties of the materials resulting in reactions at the growth and subsequent thermal treatment stages. The synthesis of perfect fianite films on Si requires a development of special methods to decrease the influence of amorphous $\mathrm{SiO}_{2}$ layer at the substrate-layer interface.

3.9. Characterization of Fianite Films. The films were studied by means of scanning electron microscopy, ellipsometry, and CV-parameters measurement techniques.

3.9.1. Capacity-Voltage Characteristic Measurements of the "Fianite-on-Silicon" Structures. The capacity-voltage (CV) characteristics of the structures supplied with fianite films deposited on $p$-Si and $n$-Si substrates were measured.

Capacity measurements provide evaluation of dielectric properties of films under the study: dielectric constant $\varepsilon$ and dielectric loss $\operatorname{tg} \delta$. The application of a multifrequency device allows the determination of frequency dependencies of dielectric constant and high-frequency loss in dielectric films. Since the dielectric film is deposited on a semiconductor, a MIS structure (metal-insulator-semiconductor) is formed, so the CV-measurement provides additional information concerning the semiconductor and the dielectricsemiconductor interface, namely, type of the semiconductor conductivity ( $n$ or $p$ ) and concentration of the dopant, flat band barrier voltage $V_{\mathrm{fb}}$, density of boundary states, and a charge induced in the dielectric.

The device used for CV-measurements allowed determining the capacity and high-frequency conductivity of the structures, as well as its dependency on the applied voltage. The measurements were carried out at $500 \mathrm{KHz}$ and $1 \mathrm{MHz}$ frequencies. Direct potential bias range was $\pm 40 \mathrm{~V}$. 


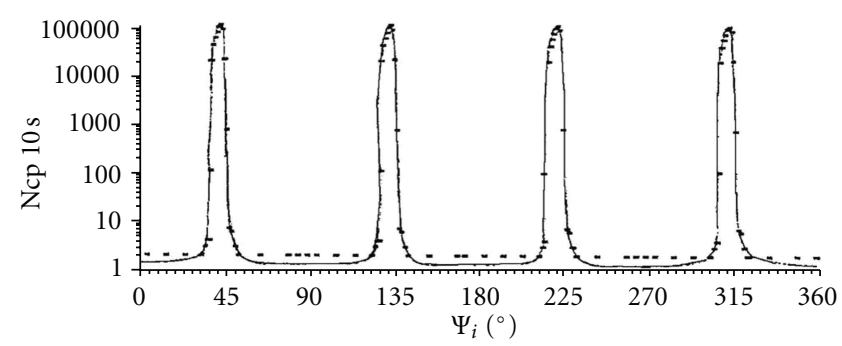

(a)

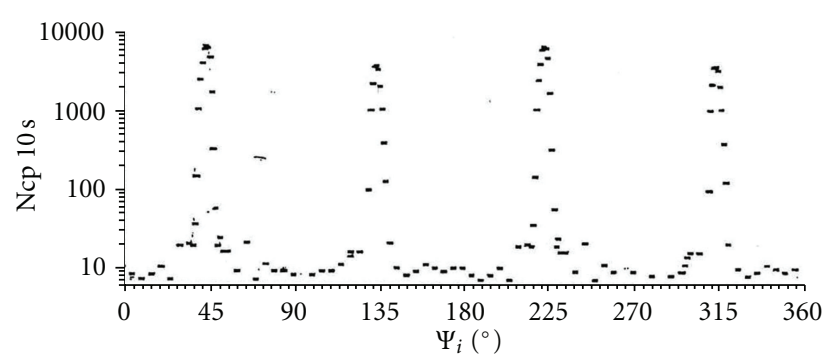

(b)

FIGURE 15: $\Psi$ scanning spectra for (422) reflection of YSZ substrate (a) and the film (b).

Thermally sputtered $\mathrm{Al}$ of $1 \mathrm{~mm}$ surface diameter was used as the contacts. The results obtained are shown in Figure 16.

"Al-fianite-Si" MIS structure parameters: flat band barrier voltage $-4 \mathrm{~V}$ for $180 \mathrm{~nm}$ film and $1.5 \mathrm{~V}$ for $20 \mathrm{~nm}$ film; density of the boundary state charge $\sim+10^{12} \mathrm{~cm}^{-2}$.

\subsubsection{Investigation of $\mathrm{ZrO}_{2}$ Films on Si and Ge Substrates by} Means of Scanning Electron Microscopy. The $\mathrm{ZrO}_{2}$ films were studied using scanning electron microscopy. All of the films studied were porous-free. Since the square of the samples studied was $5-6 \mathrm{~cm}^{2}$, it is possible to consider the porosity value at least not exceeding $0.15-0.2 \mathrm{~cm}^{-2}$. For comparison, it is worth to mention that porosity of $\mathrm{SiO}_{2}$ films is $4-8 \mathrm{~cm}^{-2}$. Therefore, it is possible to consider $\mathrm{ZrO}_{2}$ films as a protective layer for Ge devices actually superior $\mathrm{SiO}_{2}$ films because its porosity decreased in 1.5-2 orders of magnitude.

The study of morphology of the films deposited by magnetron sputtering technique at high magnification has shown its satisfactory homogeneity. Some regions of the surface featured by a relief composed by quasi-spherical hills of 500-600 $\mathrm{nm}$ in diameter and exhibiting lateral periodicity. Analytical study of the films has shown an absence of inclusions of impurities.

An attempt to study the mechanism of formation of the films with the purpose to optimize the conditions of magnetron sputtering was done using electron microscopy (JSM JEOL 5910 LV). The particles were identified by means of electron probe. The film was removed by polishing using diamond paste with $2.5-4 \mu \mathrm{m}$ particle size. This abrasive size was chosen to minimize decreasing particle size of the film constituents at the polishing. The obtained material was flushed by ethanol (9-12 purity grade "for microelectronics") and the suspension was put in plastic syringes ( $1 \mathrm{~mL})$. In order to disintegrate aggregates, ultrasonic (US) treatment was carried out. The US dispersion was conducted using "Sapphire 3 M-1.3" US device with 35 GHz operational frequency. The syringes were inserted to the device chamber filled with water. The chamber was thermostated at $27^{\circ} \mathrm{C}$. Followed by $3 \mathrm{~min}$ of the US treatment, the suspension was aspirated onto conductive (graphitized) ribbon for subsequent microscopy study. The study has shown that the largest constituents of the zirconia film were quasispherical particles of $50-100 \mathrm{~nm}$ size that explained X-ray amorphous nature of the film. It is possible to suggest

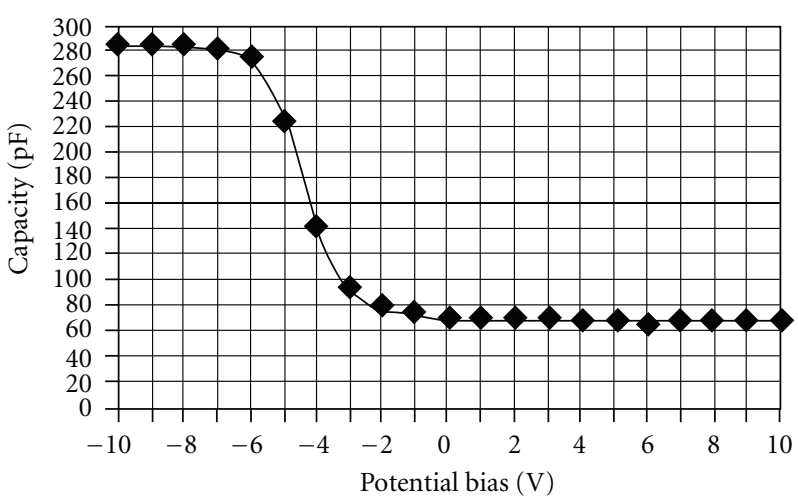

FIGURE 16: CV-characteristics of fianite-on- $p$-Si sample.

that formation of larger elements of the relief occurred by enlargement of such particles. The reasons of local enlargement (formation of spherical hills) can be gradients of temperature and mass transfer, as well as occurrence of impurities. The observations allowed refining the conditions of the sputtering of $\mathrm{ZrO}_{2}$ and fianite films in order to minimize surface roughness.

3.10. Fianite as a Gate Dielectric. Recently, a sharp surge of interest in the use of fianite as a gate dielectric in CMOS technology has been observed. It is associated with an increase of leakage current at the use of conventional $\mathrm{SiO}_{2}$ by increasing the integration level. That requires a change of $\mathrm{SiO}_{2}$ over dielectrics with higher dielectric constant (high- $k$ materials) [30, 32, 33, 45]. The resent studies have limited possible alternatives to fianite, $\mathrm{HfO}_{2}, \mathrm{ZrO}_{2}$, and its silicates. For example, $\mathrm{ZrO}_{2}$ has high dielectric constant value, good dielectric properties (5.8 eV energy gap width), and rather good crystallochemical matching with Si [50] (see Table 2). Intel Corp., one of the leaders of the world electronics, has demonstrated that the change of $\mathrm{SiO}_{2}$ over $\mathrm{HfO}_{2}$ as a gate dielectric in $45 \mathrm{~nm}$ technological process allows decreasing leakage currents, (which became a serious problem for transistors) by more than two orders of magnitude [51].

Comparison of fianite films [33] and $\mathrm{SiO}_{2}$ with electrical equivalent oxide thickness of about $1.46 \mathrm{~nm}$, has shown that the leakage current for fianite was four orders of magnitude lower than that of conventional $\mathrm{SiO}_{2}$ gate oxides. 
TABLe 3: Parameters of growth and annealing of the fianite-on-Si films.

\begin{tabular}{lcccc}
\hline Sample & $T$ of growth, C & Annealing, 600 C, 10 min & Film thickness, nm & Substrate \\
\hline z1 & Room & Without annealing & $\sim 20$ & $\sim 20$ \\
z2 & Room & Vacuum & $\sim 20$ & $\mathrm{Si}\langle\mathrm{B}\rangle$ \\
z3 & Room & Oxygen & $\sim 20$ & $\mathrm{Si}\langle\mathrm{B}\rangle$ \\
z4 & Room & Oxygen & $\sim 20$ & $\mathrm{Si}\langle\mathrm{Sb}\rangle$ \\
z5 & 600 & Oxygen & & $\mathrm{Si}\langle\mathrm{B}\rangle$ \\
\hline
\end{tabular}

The hysteresis and interface state density in this film was measured to be less than $10 \mathrm{mV}$ and $2.0 \times 10^{11} \mathrm{eV}^{-1} \mathrm{~cm}^{-2}$. Thus, such crystalline oxide on semiconductor could be used for future generation of semiconductor-based devices.

It is worth to note that quality of the synthesized fianite, as well of the interfaces, is very important for integration of such a dielectric to the CMOS technology currently in use.

Synthesizing of fianite-on-silicon structures of high quality featuring with sharp interfaces is associated with significant difficulties described above.

One of the routes to solve this problem is in application of low-temperature growth and annealing regimes, as those, which were used in the series of experiments described below, type of a substrate, and the annealing media were also varied. Conditions of the synthesis of the fianite/Si structures are given in Table 3. XRD technique has shown that fianite layers obtained by laser deposition at room temperature were of amorphous structure.

Subsequent postgrowth recrystallization annealing resulted in arising of a polycrystalline phase in the film. At the same time, the layers sustained mirror flat and uniform. Profile of the surface of $\mathrm{z} 4$ sample (Table 4) obtained using Talysurf interference microscope is shown in Figure 17(a). Roughness of this $\mathrm{ZrO}_{2}$ surface was estimated as $\mathrm{Sq}=$ $0.852 \mathrm{~nm}$ that does not practically differ from roughness of the Si substrate used for the fianite growth $(\mathrm{Sq}=0.7877 \mathrm{~nm})$.

Preliminary studies of gate properties of thin (10$15 \mathrm{~nm}$ ) fianite films obtained by laser deposition on $\mathrm{Si}$ substrates have been carried out. The studies conducted on the test structures with deposited $\mathrm{Al}$ contacts have shown that thin fianite films featured with low values of loss currents, minimum values being $10^{-12} \mathrm{~A} / \mathrm{cm}^{2}$ at $1 \mathrm{~V}$ voltage (Figure 17(b), samples z3 and z4).

\subsection{Fianite and $\mathrm{ZrO}_{2}$ as Protective and Stabilizing Layers on Ge and Si Substrates and Multilayer Structures}

3.11.1. Deposition Modes. Fianite and zirconium dioxide films were deposited by magnetron sputtering technique, 2 types of vacuum evaporation Leybold Heraeus units (Z-400 and Z-550) with different target dimensions were used: $70 \mathrm{~mm}$ in diameter for fianite and $203 \mathrm{~mm}$ - for $\mathrm{ZrO}_{2}$ (Table 4).

High-frequency and direct voltage modes of sputtering were tested. By using the latter mode it was impossible to provide sufficient film growth rate, so HF sputtering mode $(13.56 \mathrm{MHz})$ was chosen. The optimal modes of fianite and $\mathrm{ZrO}_{2}$ sputtering are also shown in Table 4. In case of low
TABLE 4: Optimal modes of fianite and $\mathrm{ZrO}_{2}$ sputtering.

\begin{tabular}{lcc}
\hline Target material & Fianite & $\mathrm{ZrO}_{2}$ \\
\hline Installation & $\mathrm{Z}-400$ & $\mathrm{Z}-550$ \\
Target diameter & $\varnothing 70 \mathrm{~mm}$ & $\varnothing 203 \mathrm{~mm}$ \\
Argon pressure & $5 * 10^{-3} \mathrm{mBar}$ & $5 * 10^{-3} \mathrm{mBar}$ \\
Power & $\sim 500 \mathrm{Wt}$ & $\sim 400 \mathrm{Wt}$ \\
Film growth rate & $100 \AA / \mathrm{min}$ & $\sim 50 \AA / \mathrm{min}$ \\
\hline
\end{tabular}

magnetron power, plasma is unstable ("blinking plasma"); in case of larger values of discharge power, the growth rate increases, but irregularity of the surface patterns and growing film coarse-graining were observed on a number of samples. Fianite sputtering requires higher power than in case of $\mathrm{ZrO}_{2}$, at the same time the growth rate was twice as much than in case of $\mathrm{ZrO}_{2}$.

The developed technique of magnetron sputtering made it possible to vary the fianite film thickness between 600 and $2000 \AA$. The optimization of the sputtering modes allowed producing of $\mathrm{Ge}$ and $\mathrm{Si}$ plates with fianite films of satisfactory quality. Ge plates with fianite films were used to try out further operations of the device structures production: photolithography and etching.

3.12. Protective and Stabilizing Properties of Fianite Films on Ge. Inorganic dielectric coatings are usually used for passivating and protection of $p-n$ transition surface, as shielding and thermal compensation layers at ion implanting and for interference antireflecting protection. Passivation of the surface is the most important issue for manufacturing of germanium photodiodes because natural $\mathrm{GeO}$ and $\mathrm{GeO}_{2}$ oxides are unstable and, so, cannot be considered as the only passivating coatings. It is one feature distinguishing $\mathrm{Ge}$ and $\mathrm{Si}$ devices (the latter have stable and rather efficient coating formed by its own $\mathrm{SiO}_{2}$ oxide). This oxide film deposited from a gas phase is of the most frequent use for photodiodes, with $p^{+}$-n-structure. It has positive charge and by attracting electrons to the surface prevents growth of $p$-channels thus decreasing probability of generation in the layer. It is worth to note that for improved reliability and stability of characteristics of photodiodes, it is necessary to maintain surface state density at $10^{11} \mathrm{~cm}^{-2} \mathrm{eV}^{-1}$ level. However, this passivating technique is far from ideal because of the high porosity of $\mathrm{SiO}_{2}$ films that decreases humidity resistance and reliability of the device.

In order to improve dielectric properties of the protective coating, fianite films deposited by magnetron sputtering were 


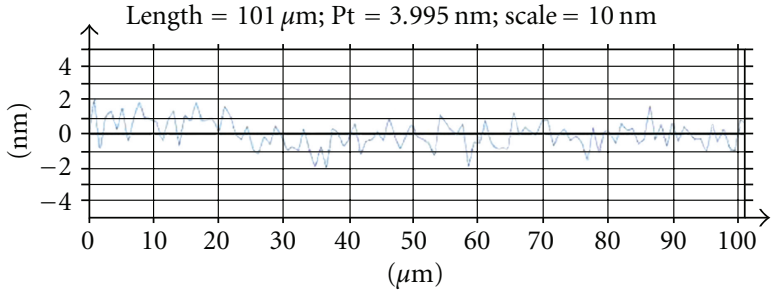

(a)

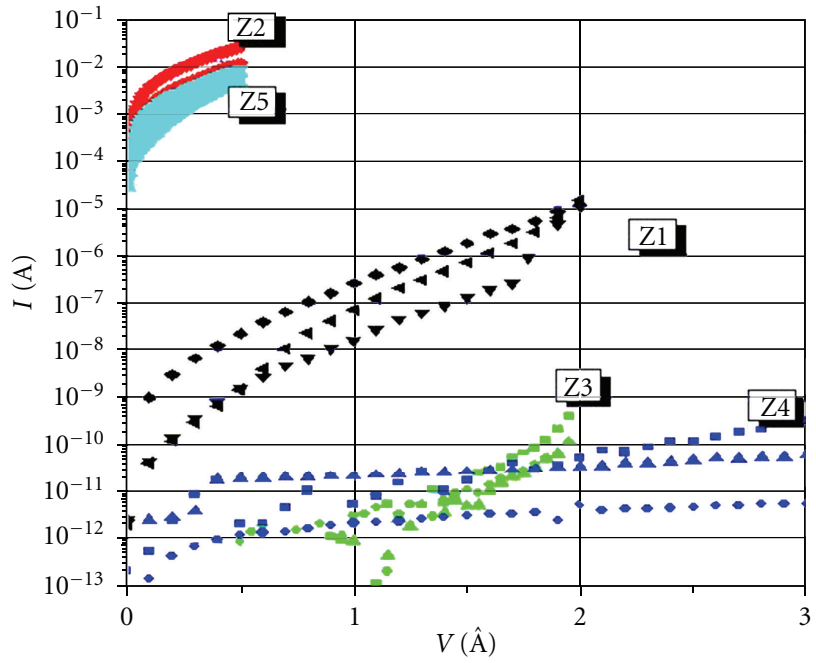

(b)

Figure 17: Surface roughness of fianite film on Si substrate, sample z4 (a); and leakage current of Al/fianite/Si structure (b) samples z1-z5 were prepared under different conditions.

used. The opportunity of its application for maintaining high-quality practically porous-free protective coating has been confirmed earlier by the experiments.

It has been demonstrated that the use of fianite protective layer in Ge-structures instead of $\mathrm{SiO}_{2}$ eliminated pulse noise and thus considerably improved photoelectric and performance characteristics of these devices. It has been established that the improvement was related to a more uniform nature of fianite films, in particular, absence of pores, in comparison with $\mathrm{SiO}_{2}$ films, which contain defects in form of pores.

3.13. Some Properties of the Device Structures Supplied with Zirconium Dioxide Films. Photoelectric characteristics and noise of germanium photodiodes supplied with $\mathrm{ZrO}_{2}$ and $\mathrm{SiO}_{2}$ films described above have been investigated. Monochromatic sensitivity of these photodiodes is typical for germanium devices and equals to $0.5-0.6 \mathrm{~A} / \mathrm{W}$ (at 1.06 and $1.55 \mu \mathrm{m}$ wavelengths). The change $\mathrm{SiO}_{2}$ over $\mathrm{ZrO}_{2}$ resulted in somewhat decrease of a dark current (on average for 10\%). Main improvement of the photodiodes quality achieved due to the application of $\mathrm{ZrO}_{2}$ films was revealed at the noise studies. Under the voltage exceeding operational one (that corresponds to accelerated reliability testing conditions), the check samples with $\mathrm{SiO}_{2}$ films have shown pulse noise of telegraphic type in the oscillogram, which can be associated with processes of energizing-deenergizing of the surface conducting channels [51]. The defects occurring because of the presence of pores in $\mathrm{SiO}_{2}$ films are probable cause of arising of the channels. In the batch with $\mathrm{ZrO}_{2}$ protective films, only shot noise, which is in principle unavoidable, was observed. More detailed results of the device studies are presented in [52].

Thus, the studies performed on fianite and zirconium dioxide films as well as on the device structures developed

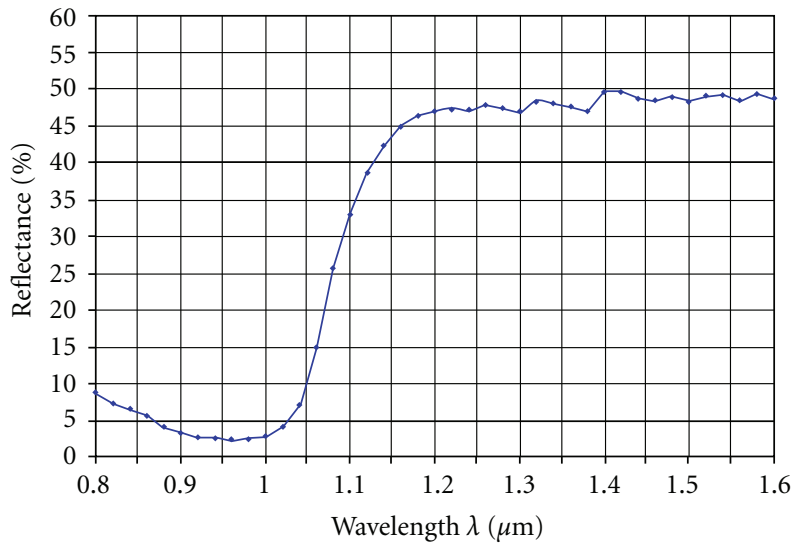

FIGURE 18: The dependency of the reflection on wavelength of silicon sample coated with $\mathrm{ZrO}_{2}$ film of $1200 \AA$ thickness.

using these films have demonstrated the advantages of zirconia-based solid solutions in application to photosensitive apparatus technology.

3.14. Studies of Optical Properties of $\mathrm{ZrO}_{2}$ Films. Optical refraction of $\mathrm{ZrO}_{2}$ equals to $1.98 \div 2.1$, that is, close to fianite one; therefore this material is also promising for antireflection coatings. Determination of the refraction constant $n$ and monitoring of the film thickness $d$ were carried out using ellipsometry technique. The experimentally determined values of $\mathrm{d}$ depended on duration of the films growth and varied within $600 \AA-1100 \AA$ range.

The films obtained have shown rather high refraction constant $\sim 2 \div 2.1$. These values were significantly higher than that of $\mathrm{SiO}_{2}$ (1.45).

In theory, considering an incident beam from air (vacuum), it is possible to decrease the reflection to zero when 


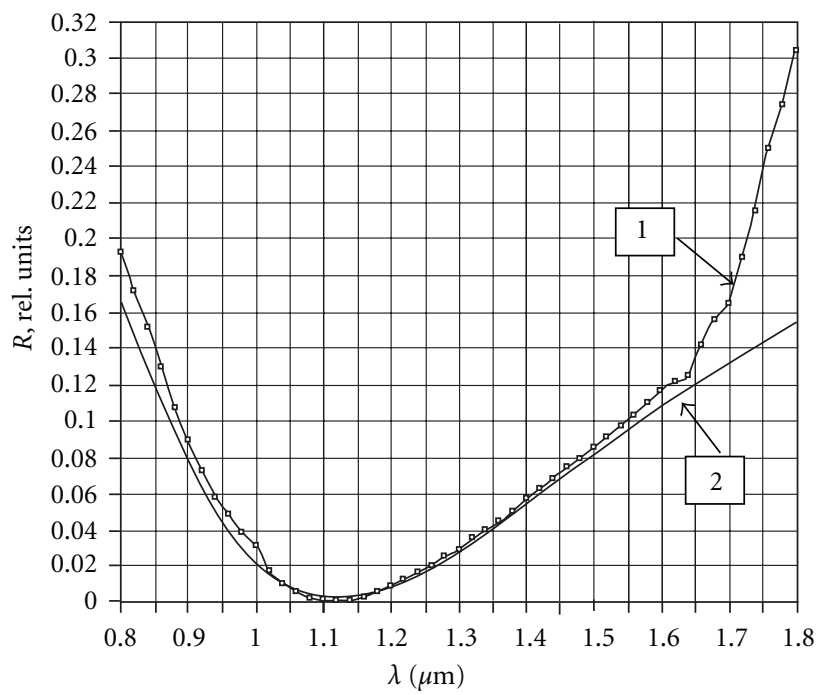

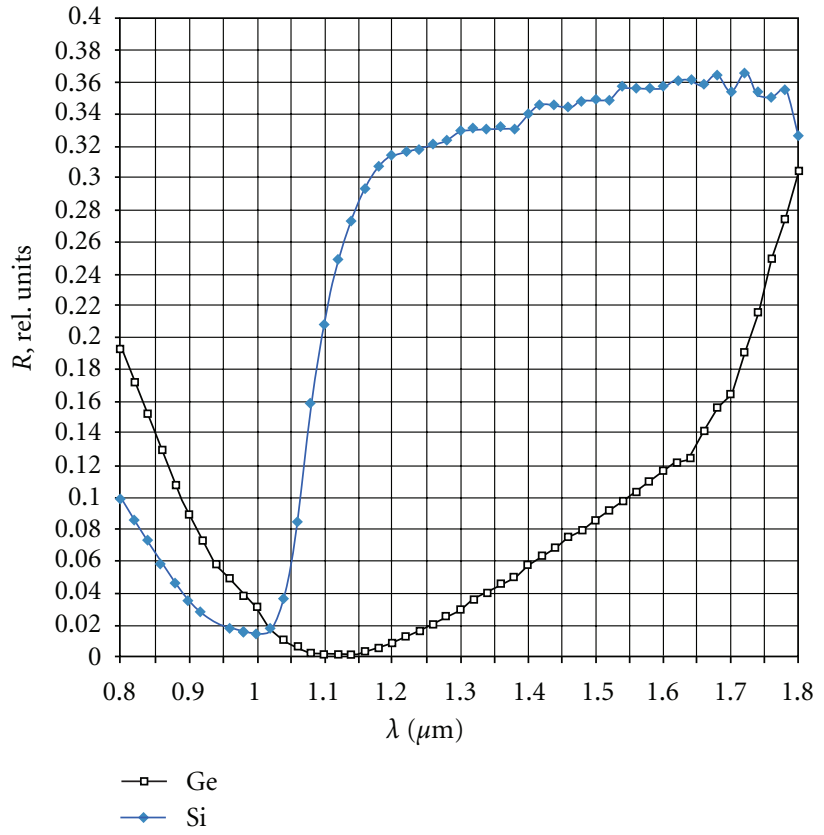

(b)

FIGURE 19: Experimental (1) and theoretical (2) dependencies of the reflection on wavelength in Ge-fianite antireflecting film system $(1300 \AA)(a)$; experimental dependencies of the reflection of fianite film on Si and Ge (b).

the refraction constant of an antireflecting film corresponds to the following equation:

$$
n=\sqrt{n_{f}}
$$

where $n_{f}$-refraction constant of a semiconductor. In case of Si and GaAs, $n_{f} \sim 3.5 \div 4$, thus $\sqrt{n_{f}} \sim 1.9 \div 2$. Therefore, $\mathrm{ZrO}_{2}$ films obtained actually satisfy perfect antireflection for Si and GaAs-based devices from the viewpoint of $n$. Moreover, the difference in $n$-values of $\mathrm{SiO}_{2}$ and $\mathrm{ZrO}_{2}$ films provides an opportunity for the antireflection over a broad spectral range due to application of binary $\mathrm{SiO}_{2}+\mathrm{ZrO}_{2}$ antireflecting coatings. The dependency of the reflection constant on wavelength of silicon sample coated with $\mathrm{ZrO}_{2}$ film of $1200 \AA$ thickness is presented in Figure 18. Theoretical absorption minimum corresponds to $\lambda=4 n \cdot d=4 \cdot 2.1$. $0.12 \approx 1 \mu \mathrm{m}$.

As it is apparent from Figure 18, the reflection minimum was approached at $\lambda_{\min }=0.97 \mu \mathrm{m}$. Thus, the experimental results are in conformity with the theory practically complete.

Therefore, $\mathrm{ZrO}_{2}$ film ensures high antireflection quality: at $\lambda_{\min }$, the reflection loss does not exceed $2-3 \%$. The data obtained confirm that $\mathrm{ZrO}_{2}$ is an excellent material for antireflecting films, as well as fianite.

\section{Fianite as Antireflecting Layer for Solar Cells}

4.1. Antireflecting Properties of Fianite Film on Ge and Si. In theory, it is possible to eliminate the reflection completely (at the corresponding thickness of a film d) at $n_{d}=\sqrt{ } n_{f}$, where $n_{f}$-optical refraction constant of a semiconductor. Since for $\mathrm{Si}$ and Ge the constants are equal to 3.7 and 4, respectively, the reflection is completely eliminated at $n_{d}=\sqrt{ } n_{f} \approx 2$. Therefore, a dielectric having its optical refraction constant $n_{d}=\sqrt{ } n_{f} \sim 2\left(\right.$ at $\left.n_{f}=3.7 \div 4\right)$ can be considered as an optimal material for the antireflecting film for solar cells and the other photosensitive devices. Theoretically, at the film thickness, which is equal to a quarter of optical wavelength $W=\lambda / 4 n_{d}$, such dielectric allows a complete elimination of the reflection loss $(R=0)$.

The refraction constant of $\mathrm{SiO}_{2}\left(n_{d}=1.47\right)$ is considerably lower than that value. At this $n$ value it is impossible to maintain the reflection loss lower than $10 \%$. Refraction constants of fianite and $\mathrm{ZrO}_{2}$ are within $(2.15 \div 2.18)$ and $(2.13 \div 2.2)$, respectively, that is close to the above optimum value. Thus, providing an evidence that fianite and $\mathrm{ZrO}_{2}$ are very promising as antireflecting coatings for solar cells and the other photosensitive devices based on $\mathrm{Ge}, \mathrm{Si}$, and $\mathrm{A}^{\mathrm{III}} \mathrm{B}^{\mathrm{V}}$ compounds.

Experimental dependencies of antireflection (as dependencies of the reflection on wavelength) of fianite films on $\mathrm{Si}$ and Ge have been plotted (Figure 19).

The plots apparently demonstrate that the reflection drops to $0-1.5 \%$ in the minima.

Experimental study of antireflective properties of fianite oxide applied to $\mathrm{Ge}$ was performed. By the reason that germanium photodetectors are designed for detecting radiation generated by lasers with wavelengths $\lambda=1.06$, 1.3 , and $1.54 \mu \mathrm{m}$, thickness of the antireflective fianite film was chosen as $W=1300 \AA$; such thickness provides for 


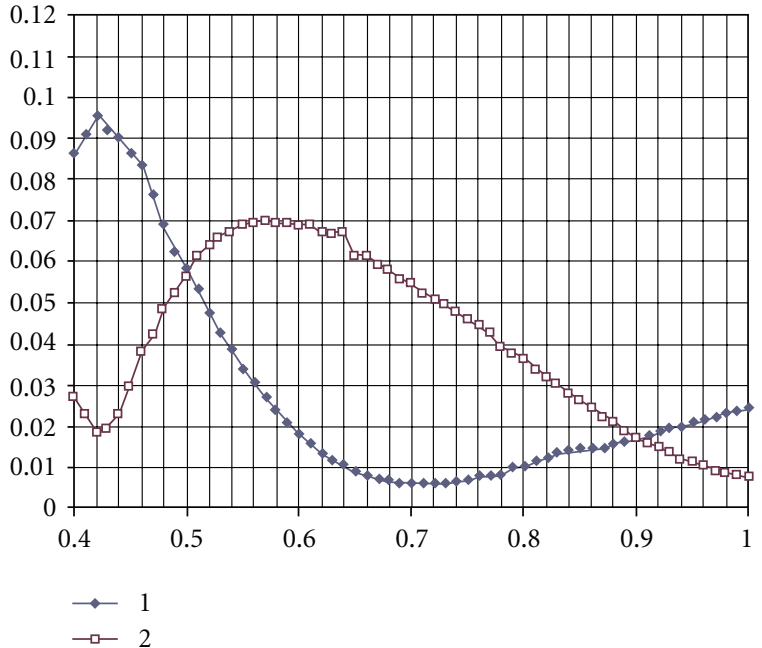

(a)

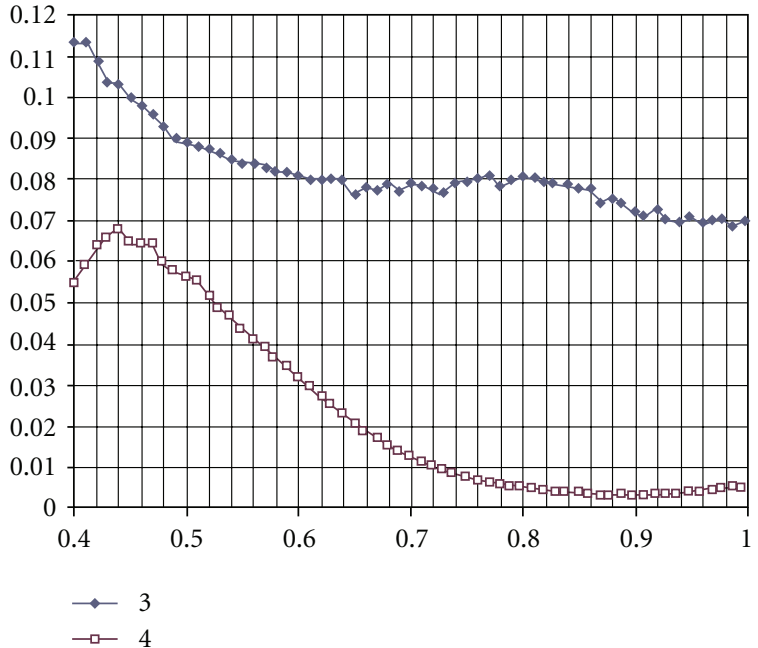

(b)

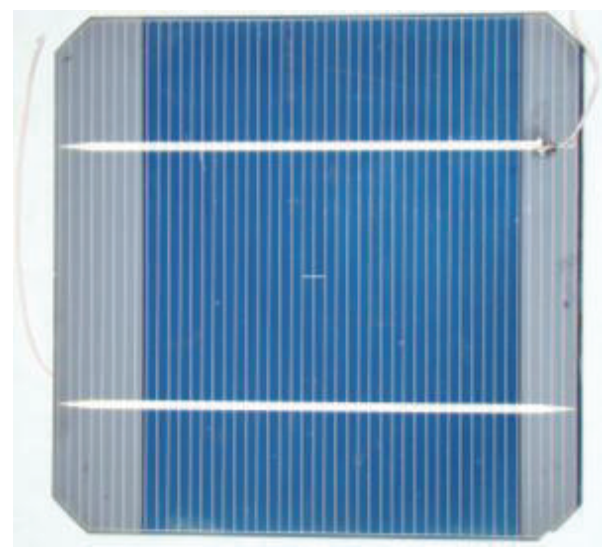

(c)

FIGURE 20: Antireflecting properties (reflection spectra) of fianite films of $580 \AA$ (a) and $1050 \AA$ (b) thickness. The experimental data were obtained on the industrial items s (c) of Si solar cells of " 4 x 4" size.

minimal reflection losses in the considered wavelength range $\lambda=1.06-1.54 \mu \mathrm{m}$. Figure 19(a) shows the comparison of experimental (thin line) and theoretical (bold line) $R(\lambda)$ curves. The theoretical $R(\lambda)$ curve was calculated using the following formula:

$$
\begin{aligned}
R=1 & -\frac{n_{f} n_{d}^{2}}{n_{d}^{2}\left(n_{f}+1\right)^{2}-\left(n_{f}^{2}-n_{d}^{2}\right)\left(n_{d}^{2}-1\right) \sin ^{2}\left(2 \pi n_{d} W / \lambda\right)} .
\end{aligned}
$$

According to the above formula, reflection may fall practically to zero at the optimal value of $n_{d}$ (note that in case of $\mathrm{SiO}_{2}$ antireflective film, for which $n_{d}=1.47$, it is impossible to obtain reflection lower than $10 \%)$. The minimal reflection is achieved at the following wavelength $\lambda_{\min }$ :

$$
\lambda_{\min }=4 W n_{d}
$$

As it is shown in Figure 19(a), in the range of fundamental absorption (for $\lambda<1.65 \mu \mathrm{m}$ ), the experimental curve 1 coincides with the theoretical curve 2. Some discrepancy at higher wavelengths $(\lambda>1.65 \mu \mathrm{m})$ appears due to deep penetration of such radiation and its reflection from the back surface. It is important that at the optimal wavelength $(\lambda=1.12 \mu \mathrm{m})$, fianite film provides for ideal antireflective properties, the reflection is actually absent. In rather large range $0.88-1.55 \mu \mathrm{m}$, into which radiation wavelengths of the most wide spread lasers fall, the losses for reflection do not exceed $10 \%$.

The experimental dependences of enlightenment (the dependence of reflectance on the wavelength) of cubic zirconia films on Si and Ge (Ge by its optical properties is similar to GaAs) exhibit excellent antireflective properties of cubic zirconia (Figure 19(b)). As is evident from the graphs, the minimum reflection can drop to $0-1,5 \%$. Position of the minimum depends on the thickness of the film. When it gets thinned twice, the minimum would be in the solar spectrum. 
Plateau in the curve shows the reflection from the back side of the substrate in the transmission range for Si. So the gain due to the use of the antireflecting fianite film reaches 20$30 \%$.

So, it was experimentally proved that for $1300 \AA$ thick fianite film, reflection may actually drop to zero in the wavelength range $\lambda=1.06-1.54 \mu \mathrm{m}$.

A new, unusual application of fianite as a reflecting film (in contrast to antireflecting!) was suggested. Such unexpected use may appear useful for screening of peripheral (nonphotosensitive) photodetector areas. Various routes of formation of photosensitive areas or metallic masks sputtered on $\mathrm{SiO}_{2}$ are currently in use for the screening. But such solution causes notable spurious capacitance of the metal-oxide-semiconductor structure; provided that such capacities are inadmissible in a number of photodetectors, in particular, in high frequency photodetectors. In case of screening by the reflecting oxide (for this purpose, the thickness should be chosen as $W=1 / 2 \lambda n_{d}$ ), no surface capacity is being formed, because spurious capacitance is absent. In such case, fianite film may reflect about $60 \%$ of radiance from the surface.

4.2. Silicon Solar Cells with Fianite Antireflecting Layers. Experimental dependencies of antireflection of fianite films deposited on commercial solar cells were recorded. The reflection spectra of fianite obtained on two such samples are shown in Figure 20. The plots (Figures 20(a) and 20(b)) demonstrate excellent antireflecting properties of the fianite films. The plots also apparently demonstrate that the reflection drops to $0-1.5 \%$ in the minima. A position of the minimum depends on the film thickness. At the film thinning the minimum occurs in solar spectrum. Therefore, energy gain due to the application of the antireflecting fianite films approaches to $20-30 \%$.

\section{Conclusions}

Due to the unique combination of physical and chemical properties, fianite is a very promising multifunctional material for novel electronic technologies. Experimental data and some theoretical considerations presented in the review demonstrate prospects of the material application either as monolithic dielectric substrate or buffer layer for heteroepitaxy of semiconductor structures and as a material for insulating, antireflecting, and protective layers for various device structures, as well as a gate dielectric. Considering the performance characteristics, fianite (cubic zirconia) was shown to be in advance in comparison with the other materials currently in use. A number of specific application fields of the material in electronics were outlined.

\section{References}

[1] V. I. Aleksandrov, V. V. Osiko, A. M. Prokhorov, and V. M. Tatarintsev, "New technique of the synthesis of refractory single crystals and molten ceramic materials," Vestnik Akademii Nauk SSSR, no. 12, pp. 29-39, 1973 (Russian).
[2] S. Yu. Kuz'minov, E. E. Lomonova, and V. V. Osiko, Cubic Zirconia and Skull Melting, Cambridge International Science, UK, 2009.

[3] V. V. Osiko, M. A. Borik, and E. E. Lomonova, "Synthesis of refractory materials by skull melting technique," in Handbook of Crystal Growth, pp. 433-469, Springer, 2010.

[4] I. Golecki, H. M. Manasevit, L. A. Moudy, J. J. Yang, and J. E. Mee, "Heteroepitaxial Si films on yttria-stabilized, cubic zirconia substrates," Applied Physics Letters, vol. 42, no. 6, pp. 501-503, 1983.

[5] D. Pribat, L. M. Mercandalli, J. Siejka, and J. Perriere, "Interface oxidation of epitaxial silicon deposits on (100) yttria stabilized cubic zirconia," Journal of Applied Physics, vol. 58, no. 1, pp. 313-320, 1985.

[6] L. M. Mercandalli, D. Diemegand, M. Crose, and Y. Sierka, "Recent progress in epitaxial growth of semiconducting materials on stabilized zirconia single crystals," Proceedings of the Society of Photo-Optical Instrumentation Engineers, vol. 623, pp. 133-210, 1986.

[7] G. Shengurov, V. N. Shabunov, A. N. Buzynin et al., "Silicon heterostructures on fianite substrates," Microelectronics, vol. 6, p. 204, 1996.

[8] A. N. Buzynin, V. V. Osiko, E. E. Lomonova, N. Yu. Buzynin, and A. S. Usikov, "Epitaxial films of GaAs and GaN on fianite substrate," in Proceedings of the Materials Research Society Symposium, vol. 512 of Wide-Bandgap Semiconductors for High Power, High Frequency and High Temperature, pp. 205-210, Pittsburg, Pa, USA, 1998.

[9] A. N. Buzynin, V. V. Osiko, K. Yu. Voronko et al., "Epitaxial $\mathrm{GaN}$ and $\mathrm{GaN}_{x} \mathrm{As}_{1-x}$ films on monolithic and porous GaAs substrates with fianit sublayer," Bulletin of the Russian Academy of Sciences: Physics, vol. 69, no. 4, pp. 557-562, 2005.

[10] P. A. Anderson, C. E. Kendrick, R. J. Kinsey et al., "(111) and (100) YSZ as substrates for indium nitride growth," Physica Status Solidi C, vol. 2, no. 7, pp. 2320-2323, 2005.

[11] S. J. Wang, C. K. Ong, S. Y. Xu et al., "Electrical properties of crystalline YSZ films on silicon as alternative gate dielectrics," Semiconductor Science and Technology, vol. 16, no. 3, pp. L13L16, 2001.

[12] S. J. Wang and C. K. Ong, "Rapid thermal annealing effect on crystalline yttria-stabilized zirconia gate dielectrics," Semiconductor Science and Technology, vol. 18, no. 2, pp. 154-157, 2003.

[13] A. N. Buzynin, V. V. Osiko, E. E. Lomonova et al., "Epitaxial films of III-V compound on fianite substrates," in Proceedings of the International Congress on Advanced Materials, Their Processes and Applications, article 672, pp. 25-28, Munich, Germany, September 2000.

[14] P. D. C. King, T. D. Veal, S. A. Hatfield et al., "X-ray photoemission spectroscopy determination of the InN/yttria stabilized cubic-zirconia valence band offset," Applied Physics Letters, vol. 91, no. 11, Article ID 112103, 3 pages, 2007.

[15] T. Nakamura, Y. Tokumoto, R. Katayama, T. Yamamoto, and K. Onabe, "RF-MBE growth and structural characterization of cubic InN films on yttria-stabilized zirconia ( $\left.\begin{array}{lll}0 & 0 & 1\end{array}\right)$ substrates," Journal of Crystal Growth, vol. 301-302, pp. 508-512, 2007.

[16] A. N. Buzynin, V. V. Osiko, Y. K. Voronko et al., "Epitaxial structures of AIIIBV materials on fianite," Izvestiya Akademii Nauk. Seriya Fizicheskaya, vol. 66, no. 9, pp. 1345-1351, 2002.

[17] A. N. Buzynin, V. V. Osiko, Y. N. Buzynin, and B. Pushnyi, "Growth on GaN and GaAs on fianite by MOCVD capillary epitaxy technique," MRS Internet Journal of Nitride Semiconductor Research, vol. 4, Article 49, 1998. 
[18] A. N. Buzynin, V. V. Osiko, E. E. Lomonova, N. Yu. Buzynin, and A. S. Usikov, "Epitaxial films of GaAs and GaN on fianite substrate," in Proceedings of the Materials Research Society Symposium, vol. 512 of Wide-Bandgap Semiconductors for High Power, High Frequency and High Temperature, pp. 205-210, Pittsburg, Pa, USA, 1998.

[19] G. G. Shahidi, "SOI technology for the GHz era," IBM Journal of Research and Development, vol. 46, no. 2-3, pp. 121-131, 2002.

[20] A. N. Buzynin, V. V. Osiko, Y. N. Buzynin et al., "Fianite: a multipurpose electronics material," Bulletin of the Russian Academy of Sciences: Physics, vol. 74, no. 7, pp. 1027-1033, 2010.

[21] R. Paszkiewicz, B. Paszkiewicz, R. Korbutowicz et al., "MOVPE GaN grown on alternative substrates," Crystal Research and Technology, vol. 36, no. 8-10, pp. 971-977, 2001.

[22] A. N. Buzynin and V. P. Kalinushkin, "Laser characterization of sapphire defectts," in Proceedings of the International Congress "Materials Week-2001" on Advanced Materials, their Processes and Applications, article 389, Munich, Germany, October 2001.

[23] A. N. Buzynin and V. P. Kalinushkin Abstracts of the, "New technique of laser characterization of sapphire and fianite crystals," in Proceedings of the Laser Interaction with Matter International Symposium (LIMIS '10), Changchun, China, August 2010.

[24] N. N. Sheftal and A. N. Buzynin, "Predominant orientation of crystals on a substrate and the effect of scratches," Vestnik Moskovskogo Universiteta, no. 3, series 4, pp. 102-104, 1972.

[25] H. I. Smith and D. C. Flanders, "Oriented crystal growth on amorphous substrates using artificial surface-relief gratings," Applied Physics Letters, vol. 32, no. 6, pp. 349-350, 1978.

[26] M. Sumiya, Y. Kurumasa, K. Ohtsuka, K. Kuwahara, Y. Takano, and S. Fuke, "Reduction of defect density in GaN epilayer having buried Ga metal by MOCVD," Journal of Crystal Growth, vol. 237-239, no. 1-4, pp. 1060-1064, 2002.

[27] E. I. Rau, A. N. Zhukov, and E. B. Yakimov, "Application of surface electron beam induced voltage method for the contactless characterization of semiconductor structures," Solid-State Phenomena, vol. 63-64, pp. 327-332, 1998.

[28] A. N. Buzynin, Y. N. Buzynin, A. V. Belyaev, A. E. Luk'yanov, and E. I. Rau, "Growth and defects of GaAs and InGaAs films on porous GaAs substrates," Thin Solid Films, vol. 515, no. 10, pp. 4445-4449, 2007.

[29] G. G. Shahidi, "SOI technology for the GHz era," IBM Journal of Research and Development, vol. 46, no. 2-3, pp. 121-131, 2002.

[30] T. Ngai, W. J. Qi, R. Sharma et al., "Electrical properties of $\mathrm{ZrO}_{2}$ gate dielectric on SiGe," Applied Physics Letters, vol. 76, no. 4, pp. 502-504, 2000.

[31] S. Abermann, G. Pozzovivo, J. Kuzmik et al., "MOCVD of $\mathrm{HfO}_{2}$ and $\mathrm{ZrO}_{2}$ high- $k$ gate dielectrics for InAlN/AlN/GaN MOS-HEMTs," Semiconductor Science and Technology, vol. 22, no. 12, pp. 1272-1275, 2007.

[32] S. J. Wang, C. K. Ong, S. Y. Xu et al., "Crystalline zirconia oxide on silicon as alternative gate dielectrics," Applied Physics Letters, vol. 78, no. 11, pp. 1604-1606, 2001.

[33] S. J. Wang, C. K. Ong, S. Y. Xu et al., "Electrical properties of crystalline YSZ films on silicon as alternative gate dielectrics," Semiconductor Science and Technology, vol. 16, no. 3, pp. L13L16, 2001.

[34] H. Fukumoto, T. Imura, and Y. Osaka, "Heteroepitaxial growth of yttria-stabilized zirconia (YSZ) on silicon," Japanese
Journal of Applied Physics, vol. 27, no. 8, pp. L1404-L1405, 1988.

[35] T. Hata, K. Sasaki, Y. Ichikawa, and K. Sasaki, "Yttria-stabilized zirconia (YSZ) heteroepitaxially grown on Si substrates by reactive sputtering," Vacuum, vol. 59, no. 2-3, pp. 381-389, 2000.

[36] A. N. Buzynin, V. V. Osiko, Y. K. Voronko et al., "Epitaxial fianit films on Si and GaAs," Bulletin of the Russian Academy of Sciences. Physics, vol. 67, no. 4, pp. 586-587, 2003.

[37] V. G. Beshenkov, A. G. Znamenskii, V. A. Marchenko, A. N. Pustovit, and A. V. Chernykh, "Widening temperature range of epitaxial growth of YSZ films on Si [100] under magnetron sputtering," Journal of Technical Physics, vol. 77, no. 5, pp. 102107, 2007.

[38] S. Jeon, M. Takanori, A. Unno, K. Wasa, Y. Ichikawa, and H. Hwang, "Interfacial properties of a hetero-structure YSZ/p-(1 0 0)Si prepared by magnetron sputtering," Vacuum, vol. 65, no. 1, pp. 19-25, 2002.

[39] N. Wakiya, T. Yamada, K. Shinozaki, and N. Mizutani, "Heteroepitaxial growth of $\mathrm{CeO}_{2}$ thin film on $\mathrm{Si}(001)$ with an ultra thin YSZ buffer layer," Thin Solid Films, vol. 371, no. 1, pp. 211-217, 2000.

[40] S. Kaneko, K. Akiyama, T. Ito et al., "Single domain epitaxial growth of yttria-stabilized zirconia on $\operatorname{Si}\left(\begin{array}{lll}1 & 1 & 1\end{array}\right)$ substrate," Ceramics International, vol. 34, no. 4, pp. 1047-1050, 2008.

[41] R. Lyonnet, A. Khodan, A. Barthélémy et al., "Pulsed laser deposition of $\mathrm{Zr}_{1-x} \mathrm{Ce}_{x} \mathrm{O}_{2}$ and $\mathrm{Ce}_{1-x} \mathrm{La}_{x} \mathrm{O}_{2-x / 2}$ for buffer layers and insulating barrier in oxide heterostructures," Journal of Electroceramics, vol. 4, no. 2, pp. 369-377, 2000.

[42] N. Pryds, B. Toftmann, J. B. Bilde-Sørensen, J. Schou, and S. Linderoth, "Thickness determination of large-area films of yttria-stabilized zirconia produced by pulsed laser deposition," Applied Surface Science, vol. 252, no. 13, pp. 4882-4885, 2006.

[43] A. P. Caricato, G. Barucca, A. Di Cristoforo et al., "Excimer pulsed laser deposition and annealing of YSZ nanometric films on Si substrates," Applied Surface Science, vol. 248, no. 1-4, pp. 270-275, 2005.

[44] S.-C. Hwang and H. S. Shin, "Effect of deposition temperature on the growth of yttria-stabilized zirconia thin films on $\mathrm{Si}(111)$ by chemical vapor deposition," Journal of the American Ceramic Society, vol. 82, no. 10, pp. 2913-2915, 1999.

[45] A. Osinsky, S. Gangopadhyay, J. W. Yang et al., "Visible-blind GaN Schottky barrier detectors grown on Si(111)," Applied Physics Letters, vol. 72, no. 5, pp. 551-553, 1998.

[46] M. F. Wu, C. Chen, D. Zhu et al., "Depth dependence of the tetragonal distortion of a GaN layer on $\mathrm{Si}(111)$ studied by Rutherford backscattering/channeling," Applied Physics Letters, vol. 80, no. 22, pp. 4130-4132, 2002.

[47] S. Guha and N. A. Bojarczuk, "Ultraviolet and violet GaN light emitting diodes on silicon," Applied Physics Letters, vol. 72, no. 4, pp. 415-417, 1998.

[48] L. Wang, X. Liu, Y. Zan et al., "Wurtzite GaN epitaxial growth on a $\mathrm{Si}(001)$ substrate using $\gamma-\mathrm{Al}_{2} \mathrm{O}_{3}$ as an intermediate layer," Applied Physics Letters, vol. 72, no. 1, pp. 109-111, 1998.

[49] N. P. Kobayashi, J. T. Kobayashi, P. D. Dapkus et al., "GaN growth on $\mathrm{Si}(111)$ substrate using oxidized AlAs as an intermediate layer," Applied Physics Letters, vol. 71, Article ID 3569, 3 pages, 1997.

[50] A. Ohtani, K. S. Stevens, and R. Beresford, "Microstructure and photoluminescence of $\mathrm{GaN}$ grown on $\mathrm{Si}(111)$ by plasmaassisted molecular beam epitaxy," Applied Physics Letters, vol. 65, no. 1, pp. 61-63, 1994. 
[51] A. J. Steckl, J. Devrajan, C. Tran, and R. A. Stall, "SiC rapid thermal carbonization of the (111)Si semiconductoron-insulator structure and subsequent metalorganic chemical vapor deposition of GaN," Applied Physics Letters, vol. 69, no. 15, pp. 2264-2266, 1996.

[52] A. N. Buzynin, T. N. Grishina, T. V. Kiselyov et al., "Zirconiabased solid solutions-New materials of photoelectronics," Optical Memory and Neural Networks, vol. 18, no. 4, pp. 312321, 2009. 

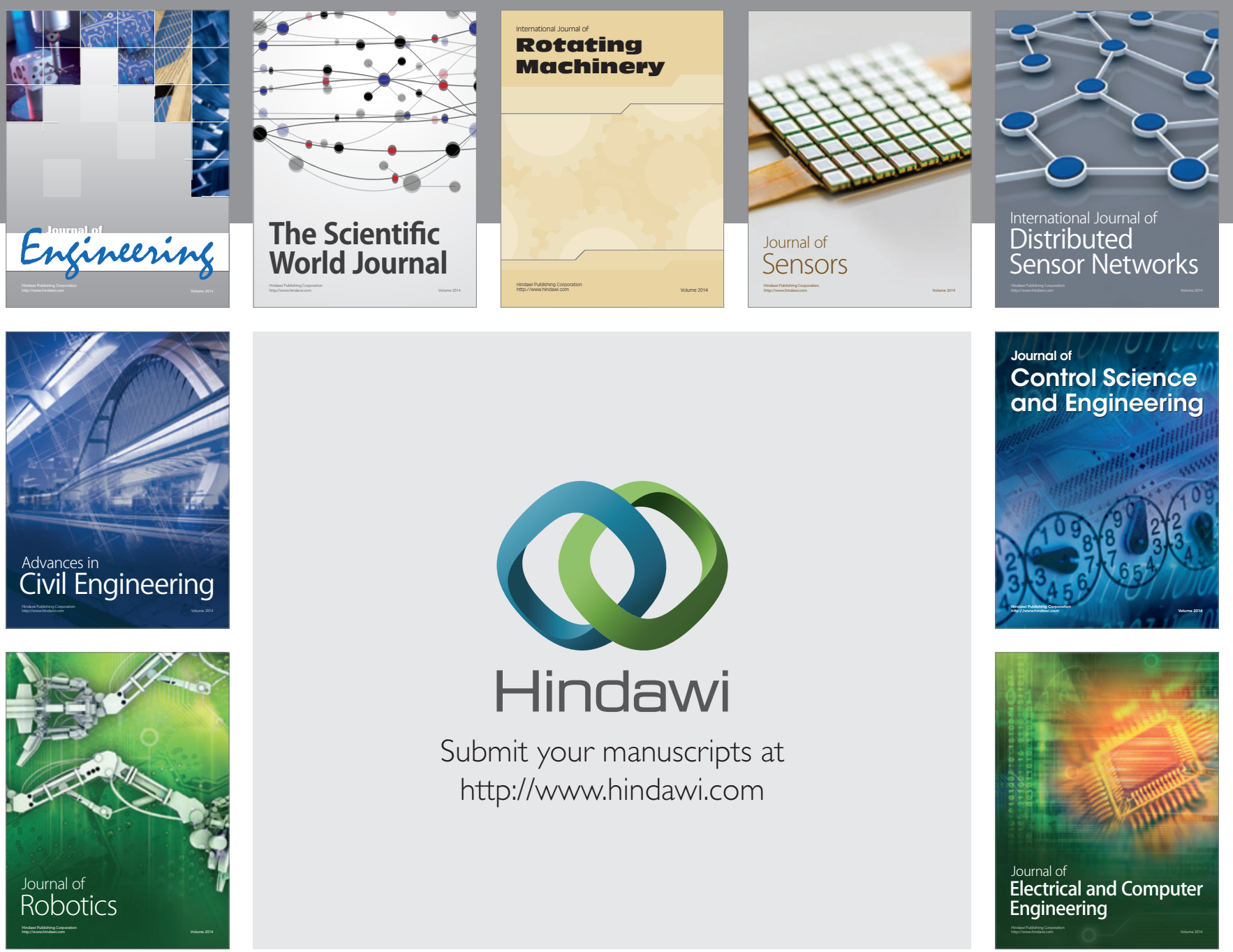

Submit your manuscripts at

http://www.hindawi.com
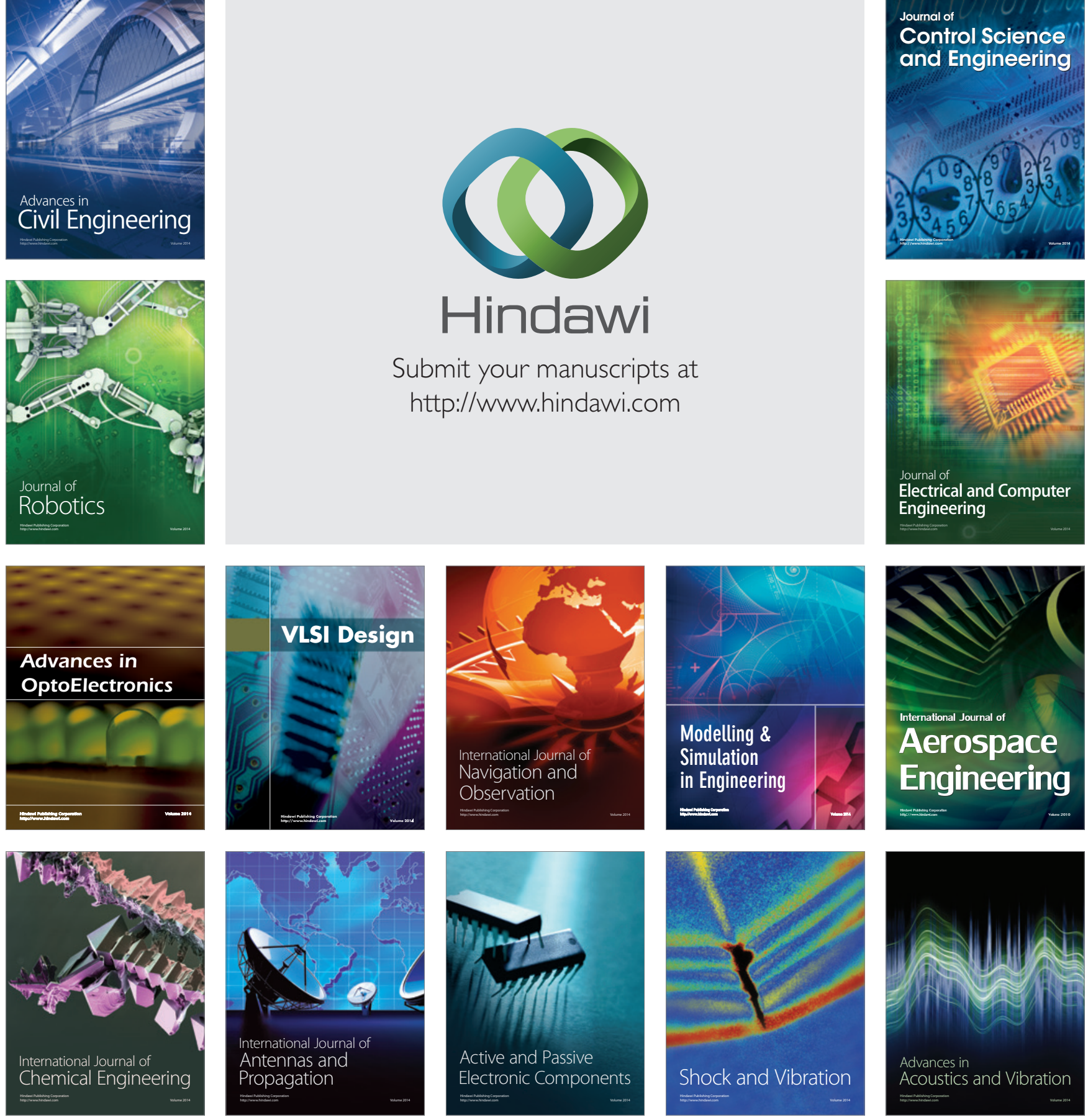